\title{
Total Synthesis of (-)-Agelastatin A
}

\author{
Takehiko Yoshimitsu,* Tatsunori Ino and Tetsuaki Tanaka* \\ Graduate School of Pharmaceutical Sciences, Osaka University, 1-6 Yamadaoka, Suita, \\ Osaka 565-0871, Japan \\ yoshimit@phs.osaka-u.ac.jp
}

\section{Supporting Information}

Table of Contents

\begin{tabular}{|c|c|c|c|}
\hline General Procedures: & $\mathrm{S} 2$ & Compound 3: & S7-8 \\
\hline Compound (+)-6: & S2-3 & Compound SI-2: & S8-9 \\
\hline Compound 9: & S3 & Compound 14: & S9-10 \\
\hline Compound 10: & S3-4 & Compound SI-3: & S11 \\
\hline Compound 11: & S4-5 & Compound 15: & $\mathrm{S} 11-12$ \\
\hline Compound SI-1: & S5 & Compound 16: & S12 \\
\hline Compound 12: & S6 & (-)-Debromoagelastatin A (17): & S13 \\
\hline Compound 5: & S6-7 & (-)-Agelastatin A (1): & S14 \\
\hline Compound 4: & S7 & ${ }^{1} \mathrm{H}$ NMR $/{ }^{13} \mathrm{C}$ NMR Spectra: & S15 \\
\hline
\end{tabular}


General Procedures. Melting points are uncorrected. Chemicals were used as received from commercial suppliers unless otherwise noted. ${ }^{1} \mathrm{H}$ NMR spectra (500, 300 or $270 \mathrm{MHz}$ ) and ${ }^{13} \mathrm{C}$ NMR spectra $(125,75$ or $67.5 \mathrm{MHz})$ were measured in $\mathrm{CDCl}_{3}$ unless otherwise stated. Chemical shifts are reported in ppm relative to the internal solvent signal: $\mathrm{CHCl}_{3}(7.26 \mathrm{ppm})$ for ${ }^{1} \mathrm{H} \mathrm{NMR}$ and $\mathrm{CDCl}_{3}$ (77.0 ppm) for ${ }^{13} \mathrm{C}$ NMR. The proton signal of TMS (0.00 ppm) or DMSO (2.50 ppm) was also used in some cases as the internal standard for ${ }^{1} \mathrm{H}$ NMR spectra. FT-IR spectra were recorded for samples loaded on $\mathrm{KBr}$ powder using the diffuse reflectance method. Mass spectra were obtained according to the specified technique. Analytical thin layer chromatography (TLC) was performed using Kieselgel $60 \mathrm{~F}_{254}$, and compounds were visualized with UV light, anisaldehyde solution, phosphomolybdic acid in $\mathrm{EtOH}$, iodine, or $\mathrm{KMnO}_{4}$ solution.

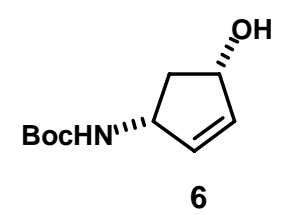

\section{(1S, 4R)-1-Hydroxy-4-aminocyclopentene (6):}

To a solution of ( $\left( \pm\right.$ )-1-hydroxy-4-aminocyclopentene (6) $(992 \mathrm{mg}, 4.98 \mathrm{mmol})$ in $\mathrm{CH}_{2} \mathrm{Cl}_{2}(30 \mathrm{~mL})$ were added vinyl acetate $(4.6 \mathrm{~mL}, 49.8 \mathrm{mmol})$ and Lipase PS-C Amano II (50 mg) at room temperature. After being stirred for $50 \mathrm{~h}$ at the same temperature, the mixture was filtered through Celite pad and the filtrate was concentrated under reduced pressure. The residue was purified by flash silica gel column chromatography (EtOAc/hexane 1:2) to give (-)-acetate 8 (490 $\mathrm{mg}, 42 \%$ ) as a colorless solid and (-)-alcohol 6 (465 mg, 48\%) as a colorless solid. The enantiomerically enriched (-)-acetate 8 obtained above ( $\sim 92 \%$ ee) was recrystallized from hexane to give enantiomerically pure (-)-acetate 8 (420 mg, 36\% overall; >99\% ee). The spectroscopic and analytical data were identical with those reported in the reference 1. This acetate (-)-8 was hydrolyzed with aq. $\mathrm{LiOH}$ in EtOH at room temperature to quantitatively yield enantiomerically pure (+)-alcohol 6. (+)-Alcohol 6: Colorless solid of mp 56-58 ${ }^{\circ} \mathrm{C}$ (EtOAc/hexane); $[\alpha]_{\mathrm{D}}{ }^{24}+66.0$ (c 1.48, $\left.\mathrm{CHCl}_{3}\right)$; IR $(\mathrm{KBr})$ v 3360, 2974, 1680 $\mathrm{cm}^{-1} ;{ }^{1} \mathrm{H}$ NMR (300 MHz, CDCl $) \delta 5.89(\mathrm{~m}, 1 \mathrm{H}), 5.77$ (m, 1H), 5.20 (brs, 1H), 4.61 (m, 1H), 4.40 (m, 1H), 3.98 (brs, 1H), 2.65 (dt, 1H, J=14.9, $7.7 \mathrm{~Hz}$ ), 1.48 (m, 1H), 1.38 (s, 9H); ${ }^{13} \mathrm{C}$ NMR (75 MHz, $\left.\mathrm{CDCl}_{3}\right) \delta 155.3,135.8,134.1,79.4,74.8,54.6,41.1,28.3$. (-)-Acetate 8: Colorless needles of mp 
57-59 ${ }^{\circ} \mathrm{C}$ (hexane) $)^{1}$; $[\alpha]_{\mathrm{D}}{ }^{24}-22.8$ (c 1.52, $\mathrm{CHCl}_{3}$ ); IR (KBr) $\vee 3366,2972,1728,1684 \mathrm{~cm}^{-1} ;{ }^{1} \mathrm{H}$ NMR (300 MHz, $\left.\mathrm{CDCl}_{3}\right) \delta 5.94$ (d, 1H, J=5.6 Hz), 5.88 (d, 1H, J=5.6 Hz), 5.48 (m, 1H), 4.76 (m, 1H), 4.54 (m, 1H), 2.78 (dt, 1H, J=14.5, $7.1 \mathrm{~Hz}$ ), 2.00 (s, 3H), 1.48 (dt, 1H, J=14.5, $4.2 \mathrm{~Hz}$ ), 1.41 (s, 9H); ${ }^{13} \mathrm{C}$ NMR (75 MHz, $\left.\mathrm{CDCl}_{3}\right) \delta 170.4,154.8,136.8,131.8,79.3,77.3,54.1,38.4,28.2,21.0$.

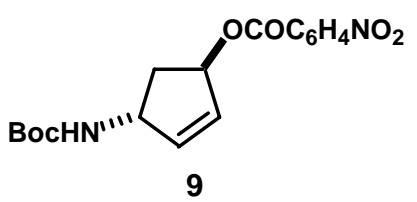

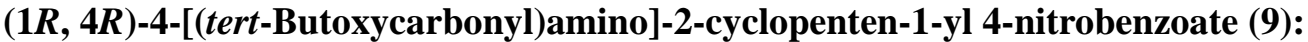

To a stirred solution of (+)-alcohol 6 (348 mg, $1.75 \mathrm{mmol})$ in THF $(20 \mathrm{~mL})$ at $-30^{\circ} \mathrm{C}$ were added $\mathrm{Ph}_{3} \mathrm{P}$ (690 mg, $2.63 \mathrm{mmol}$ ), DEAD (40\% w/v in toluene; $955 \mu \mathrm{L}, 2.1 \mathrm{mmol}$ ) and 4-nitrobenzoic acid (351 mg, $2.1 \mathrm{mmol}$ ). After $1 \mathrm{~h}$, sat $\mathrm{NaHCO}_{3}$ was added and then, the mixture was transferred to a separatory funnel where it was extracted with $\mathrm{CH}_{2} \mathrm{Cl}_{2}$. The organic phases were combined, dried over $\mathrm{MgSO}_{4}$, filtered, and concentrated under reduced pressure. The residue was purified by flash silica gel column chromatography (EtOAc/hexane 1:4) to give benzoate 9 (414 mg, 68\%) as a colorless solid. Colorless plates of mp 183-184 ${ }^{\circ} \mathrm{C}(\mathrm{EtOAc}) ;[\alpha]_{\mathrm{D}}^{25}+191$ (c 1.80, $\left.\mathrm{CHCl}_{3}\right)$; IR (KBr) $\vee 3345,2984,1713,1678$ $\mathrm{cm}^{-1}$; ${ }^{1} \mathrm{H}$ NMR (300 MHz, $\mathrm{CDCl}_{3}$ ) $\delta 8.25$ (dt, 2H, J=9.0, $2.0 \mathrm{~Hz}$ ), 8.15 (dt, 2H, J=9.0, 2.0 Hz), 6.14 (dd, 1H, J=5.7, $2.0 \mathrm{~Hz}$ ), 6.09 (dt, 1H, J=5.7, $2.0 \mathrm{~Hz}$ ), 6.02 (ddd, 1H, J=7.3, 4.7, 2.0 Hz), 4.98 (m, 1H), 4.53 (brd, 1H, $J=7.3 \mathrm{~Hz}$ ), 2.48 (ddd, 1H, $J=14.8,7.3,2.6 \mathrm{~Hz}$ ), 2.09 (ddd, 1H, $J=14.8,7.1,4.7 \mathrm{~Hz}$ ), 1.46 (s, 9H); ${ }^{13} \mathrm{C}$ NMR (75 MHz, $\mathrm{CDCl}_{3}$ ) $\delta$ 164.6, 155.4, 150.8, 139.6, 135.8, 131.6, 130.7, 123.5, 80.4, 79.8, 56.0, 39.0, 28.4; MS m/z: $349\left(\mathrm{MH}^{+}\right), 154$ (100\%); HRMS (FAB) calcd for $\mathrm{C}_{17} \mathrm{H}_{21} \mathrm{~N}_{2} \mathrm{O}_{6}$ $\left(\mathrm{MH}^{+}\right)$: 349.1399, found: 349.1393 .

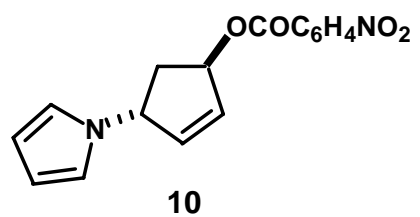

(1R, 4R)-4-(1H-Pyrroll-1-yl)-2-cyclopenten-1-yl 4-nitrobenzoate (10):

To a stirred solution of benzoate 9 (188 $\mathrm{mg}, 0.54 \mathrm{mmol})$ in $\mathrm{CH}_{2} \mathrm{Cl}_{2}(5 \mathrm{~mL})$ was added trifluoroacetic acid (360 $\mu \mathrm{L}, 4.85 \mathrm{mmol}$ ) at room temperature. After being stirred for $3 \mathrm{~h}$, the mixture was 
concentrated under reduced pressure to give crude ammonium salts that were again dissolved in $\mathrm{CH}_{2} \mathrm{Cl}_{2}(40 \mathrm{~mL})$. To this solution was added 2,5-dimethoxytetrahydrofuran (349 $\mu \mathrm{L}, 2.7 \mathrm{mmol}$ ), and the mixture was stirred at room temperature for $2.5 \mathrm{~h}$. The solution was poured into a separatory funnel where it was partitioned between sat. $\mathrm{NaHCO}_{3}$ and $\mathrm{CH}_{2} \mathrm{Cl}_{2}$. The organic extracts were combined, dried over $\mathrm{MgSO}_{4}$, filtered, and concentrated. The residue was purified by flash silica gel column chromatography (EtOAc/hexane 1:7) to give pyrrole 10 (118 mg, 74\%) as a pale yellow solid and an inseparable mixture containing pyrrole 10 (46 mg). Further chromatographic purification (EtOAc/hexane 1:12) of the mixture afforded pure pyrrole 10 (30 mg, 19\%). By this protocol, $148 \mathrm{mg}$ of pyrrole 10 (92\%) was obtained in total. Pale yellow needles of mp $83{ }^{\circ} \mathrm{C}\left(\mathrm{Et}_{2} \mathrm{O}\right)$; $[\alpha]_{\mathrm{D}}{ }^{23}+317$ (c 1.51, $\mathrm{CHCl}_{3}$ ); IR (KBr) v 3102, $1732 \mathrm{~cm}^{-1} ;{ }^{1} \mathrm{H}$ NMR $\left(300 \mathrm{MHz}, \mathrm{CDCl}_{3}\right) \delta 8.30$ (dt, 2H, J=9.0, $\left.2.0 \mathrm{~Hz}\right), 8.20$ (dt, 2H, J=9.0, 2.0 Hz), 6.69 (t, 2H, J=2.0 Hz), 6.30 (m, 2H), 6.13-6.21 (m, 3H), 5.46 (m, 1H), 2.62 (ddd, 1H, $J=15.0,7.7,2.6 \mathrm{~Hz}$ ), 2.42 (ddd, $1 \mathrm{H}, J=15.0,7.3,4.6 \mathrm{~Hz}) ;{ }^{13} \mathrm{C}$ NMR $\left(75 \mathrm{MHz}, \mathrm{CDCl}_{3}\right) \delta$ 164.2, 150.3, 138.1, 135.2, 132.7, 130.5, 123.3, 118.6, 108.5, 80.3, 63.5, 39.9; MS m/z: $299\left(\mathrm{MH}^{+}\right)$, 154 (100\%); HRMS (FAB) calcd for $\mathrm{C}_{16} \mathrm{H}_{15} \mathrm{~N}_{2} \mathrm{O}_{4}\left(\mathrm{MH}^{+}\right)$: 299.1032, found: 299.1035.

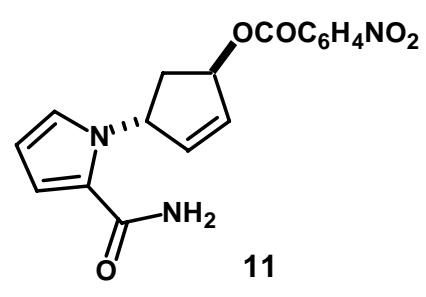

(1R,4R)-4-(2-Carbamoyl-1H-pyrrol-1-yl)cyclopent-2-en-1-yl 4-nitrobenzoate (11)

To a stirred solution of ester $\mathbf{1 0}$ (966 mg, $3.24 \mathrm{mmol})$ in $\mathrm{CH}_{2} \mathrm{Cl}_{2}(10 \mathrm{~mL})$ was added trichloroacetyl isocyanate (772 $\mu \mathrm{L}, 6.48 \mathrm{mmol})$ at room temperature. After $2 \mathrm{~h}$, the mixture was concentrated under reduced pressure. The residue was dissolved in $\mathrm{MeOH}(5 \mathrm{~mL})$, followed by adding AcOH (2 mL), and the mixture was stirred at room temperature for further $21 \mathrm{~h}$. After concentration of the mixture under reduced pressure, the residue was transferred to a separatory funnel where it was partitioned between EtOAc and sat. $\mathrm{NaHCO}_{3}$. The organic extracts were combined, dried over $\mathrm{MgSO}_{4}$, filtered, and concentrated. The residue was purified by flash silica gel column chromatography (EtOAc/hexane 1:2) to give amide 11 (1.1 g, quant.) as a pale yellow oil. Pale yellow oil; $[\alpha]_{\mathrm{D}}^{25}+202$ (c 1.50, $\mathrm{CHCl}_{3}$ ); IR $(\mathrm{KBr}) \vee$ 3356, 3188, 1721, $1651 \mathrm{~cm}^{-1}$; ${ }^{1} \mathrm{H}$ NMR $\left(300 \mathrm{MHz}, \mathrm{CDCl}_{3}\right) \delta 8.28$ (dt, 2H, J=8.8, $\left.2.0 \mathrm{~Hz}\right), 8.20$ 
(dt, 2H, $J=8.8,2.0 \mathrm{~Hz}$ ), 6.82 (dd, 1H, $J=2.7,1.7 \mathrm{~Hz}$ ), 6.71-6.64 (m, 2H), 6.33-6.26 (m, 2H), 6.17-6.09 (m, 2H), 5.59 (brs, 2H), 2.78 (ddd, $1 \mathrm{H}, J=15.1,7.8,2.6 \mathrm{~Hz}$ ), 2.26 (ddd, $1 \mathrm{H}, J=15.1,7.3,4.5 \mathrm{~Hz}$ ); ${ }^{13} \mathrm{C}$ NMR (75 MHz, $\left.\mathrm{CDCl}_{3}\right) \delta 164.6,163.5,150.5,138.0,135.4,133.2,130.8,124.2,123.5,123.2,113.8$, 108.3, 80.4, 62.0, 40.2; MS m/z: $341\left(\mathrm{M}^{+}\right)$, 174 (100\%); HRMS (EI) calcd for $\mathrm{C}_{17} \mathrm{H}_{15} \mathrm{~N}_{3} \mathrm{O}_{5}\left(\mathrm{M}^{+}\right)$: 341.1011, found: 341.1010

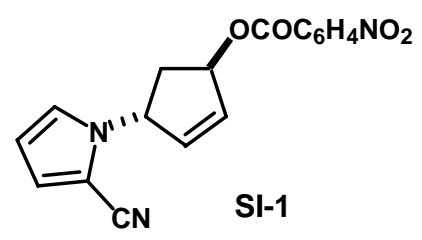

(1R,4R)-4-(2-Cyano-1H-pyrrol-1-yl)cyclopent-2-en-1-yl 4-nitrobenzoate (SI-1)

To a stirred solution of DMF (112 $\mu \mathrm{L}, 1.44 \mathrm{mmol})$ in MeCN $(2 \mathrm{~mL})$ was added $\mathrm{POCl}_{3}(135 \mu \mathrm{L}, 1.44$ $\mathrm{mmol})$ at $0^{\circ} \mathrm{C}$. After $5 \mathrm{~min}$, a solution of amide $\mathbf{1 1}$ (246 mg, $\left.0.722 \mathrm{mmol}\right)$ in $\mathrm{MeCN}(2 \mathrm{~mL}+1 \mathrm{~mL}$ used for rinsing) was added slowly to the solution by means of a cannula, and then pyridine (126 $\mu \mathrm{L}, 1.59$ mmol) was added. After $10 \mathrm{~min}$, the mixture was carefully neutralized with $1 \mathrm{~N} \mathrm{HCl}$, transferred to a separatory funnel and extracted with $\mathrm{Et}_{2} \mathrm{O}$. The organic extracts were combined, dried over $\mathrm{MgSO}_{4}$, filtered, and concentrated to give sufficiently pure nitrile (SI-1; 233 mg, quant.) as a colorless solid. This material was used for the next reaction without purification. The spectroscopic and analytical data shown below were those obtained for a partially purified material by recrystallization from EtOAc. Colorless cubes of mp 96-97 ${ }^{\circ} \mathrm{C}(\mathrm{EtOAc}) ;[\alpha]_{\mathrm{D}}{ }^{25}+218$ (c 1.00, $\left.\mathrm{CHCl}_{3}\right)$; IR (KBr) v 3113, 2214, 1724 $\mathrm{cm}^{-1} ;{ }^{1} \mathrm{H}$ NMR (300 MHz, $\mathrm{CDCl}_{3}$ ) $\delta 8.29$ (dt, 2H, J=9.0, $2.0 \mathrm{~Hz}$ ), 8.20 (dt, 2H, J=9.0, $2.0 \mathrm{~Hz}$ ), 6.83 (dd, 1H, J=3.9, $1.6 \mathrm{~Hz}$ ), 6.81 (dd, 1H, J=2.7, $1.6 \mathrm{~Hz}$ ), 6.42 (dt, 1H, J=5.6, $2.1 \mathrm{~Hz}$ ), 6.25 (ddd, 1H, $J=5.6,2.1,0.9 \mathrm{~Hz}$ ), 6.22 (dd, 1H, $J=3.9,2.7 \mathrm{~Hz}$ ), 6.17 (m, 1H), 5.72 (m, 1H), 2.74 (ddd, 1H, J=15.1, 7.8, $2.6 \mathrm{~Hz}$ ), 2.40 (ddd, $1 \mathrm{H}, J=15.1,7.2,4.6 \mathrm{~Hz}) ;{ }^{13} \mathrm{C} \mathrm{NMR}\left(75 \mathrm{MHz}, \mathrm{CDCl}_{3}\right.$ ) $\delta$ 164.2, 150.4, 136.0, 135.0, 134.7, 130.6, 123.3, 123.2, 120.4, 113.4, 109.9, 103.2, 79.8, 62.8, 39.3; MS m/z: $323\left(\mathrm{M}^{+}\right), 150$ (100\%); HRMS (EI) calcd for $\mathrm{C}_{17} \mathrm{H}_{13} \mathrm{~N}_{3} \mathrm{O}_{4}\left(\mathrm{M}^{+}\right)$: 323.0906, found: 323.0910.

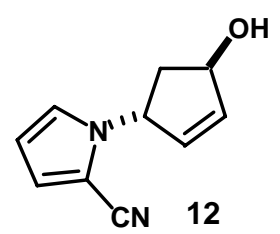




\section{1-[(1R,4R)-4-Hydroxycyclopent-2-en-1-yl]-1H-pyrrole-2-carbonitrile (12)}

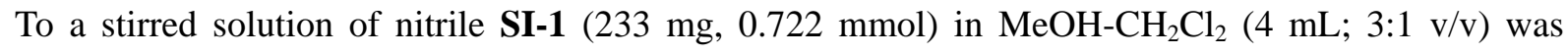
added $\mathrm{K}_{2} \mathrm{CO}_{3}\left(299 \mathrm{mg}, 2.17 \mathrm{mmol}\right.$ ) at room temperature. After $10 \mathrm{~min}$ of stirring, sat. $\mathrm{NH}_{4} \mathrm{Cl}$ was added to the solution and the mixture was concentrated under reduced pressure. The residue was transferred to a separatory funnel and extracted with $\mathrm{CH}_{2} \mathrm{Cl}_{2}$. The organic extracts were combined, dried over $\mathrm{MgSO}_{4}$, filtered, and concentrated. The residue was purified by flash silica gel column chromatography (EtOAc/hexane 2:5) to give alcohol 12 (110 mg, 88\%) as a colorless oil. Colorless oil; $[\alpha]_{\mathrm{D}}{ }^{29}+120$ (c 1.16, $\mathrm{CHCl}_{3}$ ); IR (neat) $v$ 3412, 3121, $2216 \mathrm{~cm}^{-1} ;{ }^{1} \mathrm{H}$ NMR $\left(300 \mathrm{MHz}, \mathrm{CDCl}_{3}\right) \delta$ 6.80 (dd, 1H, J=3.9, 1.6 Hz), 6.75 (dd, 1H, J=2.8, 1.6 Hz), 6.26 (dt, 1H, J=5.6, 2.0 Hz), 6.15 (dd, 1H, $J=3.9,2.8 \mathrm{~Hz}$ ), 6.02 (ddd, 1H, $J=5.6,2.2,1.0 \mathrm{~Hz}$ ), 5.61 (m, 1H), 5.15 (m, 1H), 2.43 (ddd, 1H, $J=14.7$, 7.9, $3.1 \mathrm{~Hz}$ ), 2.31 (brs, 1H), 2.22 (ddd, $1 \mathrm{H}, J=14.7,7.0,4.3 \mathrm{~Hz}$ ); ${ }^{13} \mathrm{C} \mathrm{NMR}\left(67.5 \mathrm{MHz}, \mathrm{CDCl}_{3}\right) \delta 139.4$, 132.4, 123.5, 120.5, 113.6, 109.6, 102.8, 75.8, 63.4, 42.6; MS m/z: $174\left(\mathrm{M}^{+}\right)$, 92 (100\%); HRMS (EI) calcd for $\mathrm{C}_{10} \mathrm{H}_{10} \mathrm{~N}_{2} \mathrm{O}\left(\mathrm{M}^{+}\right)$: 174.0793, found: 174.0799.

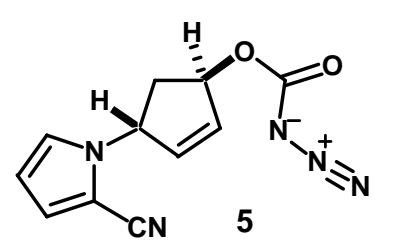

\section{1-[(1R,4R)-4-[(Azidocarbonyl)oxy]cyclopent-2-en-1-yl]-1H-pyrrole-2-carbonitrile (5)}

To a solution of alcohol 12 (724 mg, $4.16 \mathrm{mmol})$ in THF (15 mL) was added 1,1'-carbonyldiimidazole ( $2.02 \mathrm{~g}, 12.5 \mathrm{mmol}$ ) at room temperature, and the mixture was stirred for $5 \mathrm{~h}$. To this solution were added trimethylsilyl azide $(5.45 \mathrm{~mL}, 41.6 \mathrm{mmol})$, EtOAc $(15 \mathrm{~mL})$ and water $(15 \mathrm{~mL})$ at room temperature. After being stirred for $3 \mathrm{~h}$, the mixture was transferred to a separatory funnel and extracted with EtOAc. The organic extracts were combined and immediately filtered through silica gel pad to remove remaining imidazole. Then the filtrate was concentrated under reduced pressure to give the residue, which was purified by flash silica gel column chromatography (EtOAc/hexane 1:6) to provide azidoformate 5 (978 mg, 97\%) as a colorless oil. Colorless oil; $[\alpha]_{\mathrm{D}}^{28}+164\left(c \mathrm{1.44}, \mathrm{CHCl}_{3}\right)$; IR $(\mathrm{KBr}) \vee 3125,2218,1728 \mathrm{~cm}^{-1}$; ${ }^{1} \mathrm{H}$ NMR $\left(300 \mathrm{MHz}, \mathrm{CDCl}_{3}\right) \delta 6.82$ (dd, $\left.1 \mathrm{H}, J=4.0,1.6 \mathrm{~Hz}\right), 6.76$ (dd, 1H, $J=2.7,1.6 \mathrm{~Hz}$ ), 6.31 (dt, 1H, $J=5.7,2.2 \mathrm{~Hz}$ ), 6.23 (dd, 1H, $J=5.7,2.0 \mathrm{~Hz}$ ), 6.19 (dd, 1H, $J=4.6$, 
$2.7 \mathrm{~Hz}$ ), 5.92 (m, 1H), 5.64 (m, 1H), 2.65 (ddd, 1H, J=15.2, 7.7, 2.4 Hz), 2.29 (ddd, 1H, J=15.2, 7.1, 4.6 Hz); ${ }^{13} \mathrm{C}$ NMR (75 MHz, $\left.\mathrm{CDCl}_{3}\right) \delta 157.3,136.9,134.1,123.3,120.7,113.4,110.1,103.3,82.5$, 62.8, 39.2; MS m/z: $243\left(\mathrm{M}^{+}\right), 157$ (100\%); HRMS (EI) calcd for $\mathrm{C}_{11} \mathrm{H}_{9} \mathrm{~N}_{5} \mathrm{O}_{2}\left(\mathrm{M}^{+}\right)$: 243.0756, found: 243.0741.

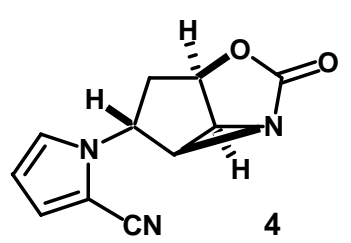

1-[(1S,5R,7R,8R)-3-Oxo-4-oxa-2-azatricyclo[3.3.0.0 $\left.{ }^{2,8}\right]$ octan-7-yl]-1H-pyrrole-2-carbonitrile (4)

In a high-pressure stainless steel tube were placed azidoformate 5 (447 mg, $1.84 \mathrm{mmol}$ ) and $\mathrm{CH}_{2} \mathrm{Cl}_{2}$ (50 mL) under an argon atmosphere. The solution was heated at $160^{\circ} \mathrm{C}$ for $13 \mathrm{~h}$ in an oil bath. The solvent was removed under reduced pressure to give the residue, which was purified by flash silica gel column chromatography (EtOAc/hexane 1:2) to afford aziridine 4 (367 mg, 92\%) as a colorless oil. Colorless oil; $[\alpha]_{\mathrm{D}}{ }^{26}+32.5$ (c 1.57, $\mathrm{CHCl}_{3}$ ); IR (neat) $v$ 3123, 2216, $1780 \mathrm{~cm}^{-1}$; ${ }^{1} \mathrm{H}$ NMR $(300 \mathrm{MHz}$, $\mathrm{CDCl}_{3}$ ) $\delta 6.93(\mathrm{~m}, 2 \mathrm{H}), 6.24$ (dd, 1H, $J=3.9,2.7 \mathrm{~Hz}$ ), 5.21 (t, 1H, $J=3.5 \mathrm{~Hz}$ ), 4.92 (dd, 1H, $J=9.0,6.4$ Hz), 4.19 (dd, 1H, J=3.5, 2.8 Hz), 3.53 (d, 1H, J=2.8 Hz), 2.90 (dd, 1H, J=14.0, 6.4 Hz), 2.75 (ddd, 1H, J=14.0, 9.0, $3.5 \mathrm{~Hz}) ;{ }^{13} \mathrm{C}$ NMR (75 MHz, $\mathrm{CDCl}_{3}$ ) $\delta 163.1$, 126.8, 122.7, 113.5, 110.2, 101.7, 81.2, 56.9, 55.7, 53.4, 49.0; MS m/z: $238\left(\mathrm{MNa}^{+}\right)$, 238 (100\%); HRMS (FAB) calcd for $\mathrm{C}_{11} \mathrm{H}_{10} \mathrm{~N}_{3} \mathrm{O}_{2}\left(\mathrm{MH}^{+}\right)$: 216.0773, found: 216.0773 .

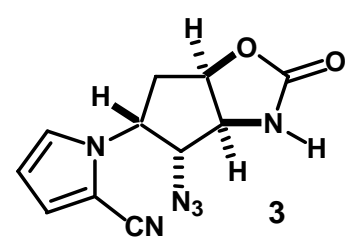

1-[(3aS,5R,6aR)-4-Azido-2-oxo-hexahydro-2H-cyclopenta[d][1,3]oxazol-5-yl]-1H-pyrrole-2-carbo nitrile (3)

To a solution of aziridine 4 (266 mg, $1.24 \mathrm{mmol})$ in DMF (15 mL) was added $\mathrm{NaN}_{3}$ (121 mg, 1.85 mmol) at room temperature. After 30 min, water was added and the mixture was extracted with EtOAc. The organic extracts were combined, dried over $\mathrm{MgSO}_{4}$, filtered, and concentrated. The residue was 
purified by flash silica gel column chromatography $\left(\mathrm{Et}_{2} \mathrm{O}\right)$ to give azide 3 (196 mg, 61\%) as a colorless solid. Colorless needles of mp $118{ }^{\circ} \mathrm{C}$ (EtOAc/hexane); $[\alpha]_{\mathrm{D}}{ }^{25}+68.4$ (c 1.27, $\mathrm{CHCl}_{3}$ ); IR $(\mathrm{KBr}) \vee$ 3350, 2955, 2216, 2110, $1755 \mathrm{~cm}^{-1}$; ${ }^{1} \mathrm{H}$ NMR (500 MHz, CDCl $) \delta 6.99$ (dd, 1H, J=2.7, 1.5 Hz), 6.90 (dd, 1H, J=4.0, $1.5 \mathrm{~Hz}$ ), 6.81 (brs, 1H), 6.28 (dd, 1H, $J=4.0,2.7 \mathrm{~Hz}$ ), 5.21 (dd, 1H, $J=7.6$, $5.2 \mathrm{~Hz}$ ), 5.15 (ddd, 1H, J=12.5, 7.0, $4.3 \mathrm{~Hz}$ ), 4.30 (dd, 1H, J=7.6, $0.9 \mathrm{~Hz}$ ), 4.27 (d, 1H, J=4.3 Hz), 2.66-2.54 (m, 2H); ${ }^{13} \mathrm{C}$ NMR (125 MHz, $\left.\mathrm{CDCl}_{3}\right) \delta 158.9,124.8,121.5$, 113.6, 110.2, 104.2, 77.5, 68.2, 60.3, 57.6, 34.1; MS m/z: $258\left(\mathrm{M}^{+}\right), 118(100 \%)$; HRMS (EI) calcd for $\mathrm{C}_{11} \mathrm{H}_{10} \mathrm{~N}_{6} \mathrm{O}_{2}\left(\mathrm{M}^{+}\right)$: 258.0865, found: 258.0863 .

\section{Synthesis of lactam 14 from azide 3}
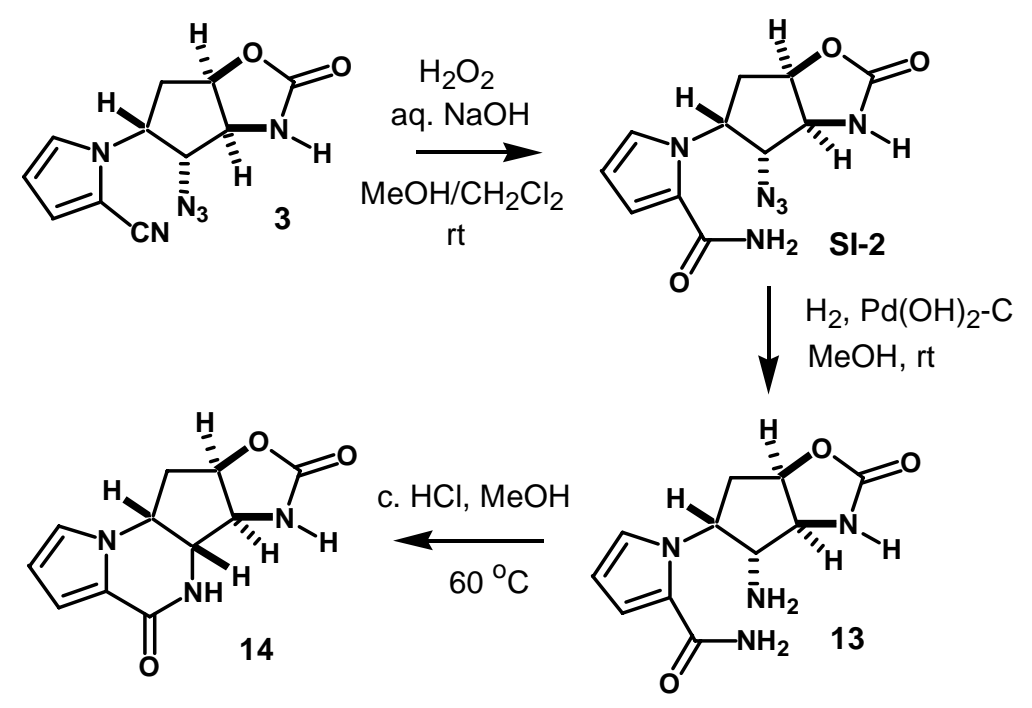<smiles>NC(=O)c1cccn1[C@H]1C[C@@H]2OC(=O)N[C@H]2[C@H]1N</smiles>

\section{1-[(3aS,4S,5R,6aR)-4-Azido-2-oxo-hexahydro-2H-cyclopenta[d][1,3]oxazol-5-yl]-1H-pyrrole-2-ca}

\section{rboxamide (SI-2)}

To a solution of azide 3 (146 mg, $0.566 \mathrm{mmol})$ in $\mathrm{MeOH}-\mathrm{CH}_{2} \mathrm{Cl}_{2}(11 \mathrm{~mL} ; 10: 1 \mathrm{v} / \mathrm{v})$ were added $1 \mathrm{~N}$ $\mathrm{NaOH}(1 \mathrm{~mL})$ and $30 \% \mathrm{H}_{2} \mathrm{O}_{2}(1 \mathrm{~mL})$ at room temperature. The reaction mixture was stirred at the same temperature for $18 \mathrm{~h}$, and then to this solution were added sat. $\mathrm{Na}_{2} \mathrm{~S}_{2} \mathrm{O}_{3}$ and sat. $\mathrm{NH}_{4} \mathrm{Cl}$. After concentration of the mixture under reduced pressure, the residue was diluted with $\mathrm{CH}_{2} \mathrm{Cl}_{2}$, transferred to a separatory funnel where it was partitioned between $\mathrm{CH}_{2} \mathrm{Cl}_{2}$ and water. The organic extracts were 
combined, dried over $\mathrm{MgSO}_{4}$, filtered, and concentrated to give sufficiently pure amide SI-2 (151 mg, 97\%) as a colorless solid. This material was used for the next reaction without purification. Colorless prisms of mp 111-114 ${ }^{\circ} \mathrm{C}$ (EtOAc/hexane); $[\alpha]_{\mathrm{D}}{ }^{27}+58.8$ (c 1.13, $\mathrm{CHCl}_{3}$ ); IR (neat) v 3335, 2926, 2106, 1746, $1649 \mathrm{~cm}^{-1} ;{ }^{1} \mathrm{H}$ NMR (300 MHz, $\mathrm{CDCl}_{3}$ ) $\delta 7.00$ (dd, 1H, J=2.7, 1.6 Hz), 6.85 (brs, 1H), 6.75 (dd, 1H, $J=3.9,1.6 \mathrm{~Hz}$ ), 6.24 (dd, 1H, $J=3.9,2.7 \mathrm{~Hz}$ ), 6.04 (brs, 2H), 5.84 (ddd, 1H, $J=13.5,6.0,4.0 \mathrm{~Hz}$ ), 5.14 (dd, 1H, J=7.5, $6.2 \mathrm{~Hz}$ ), 4.56 (d, 1H, $J=4.0 \mathrm{~Hz}$ ), 4.20 (d, 1H, J=7.5 Hz), 2.56 (td, 1H, $J=13.5,6.0$ $\mathrm{Hz}), 2.40$ (dd, $1 \mathrm{H}, J=13.5,6.2 \mathrm{~Hz}) ;{ }^{13} \mathrm{C}$ NMR $\left(75 \mathrm{MHz}, \mathrm{CDCl}_{3}\right) \delta 164.2,159.4,124.8,124.6,115.2$, 108.6, 68.5, 60.0, 56.8, 53.4, 33.8; MS m/z: $276\left(\mathrm{M}^{+}\right)$, 94 (100\%); HRMS (EI) calcd for $\mathrm{C}_{11} \mathrm{H}_{12} \mathrm{~N}_{6} \mathrm{O}_{3}$ $\left(\mathrm{M}^{+}\right)$: 276.0970, found: 276.0970 .
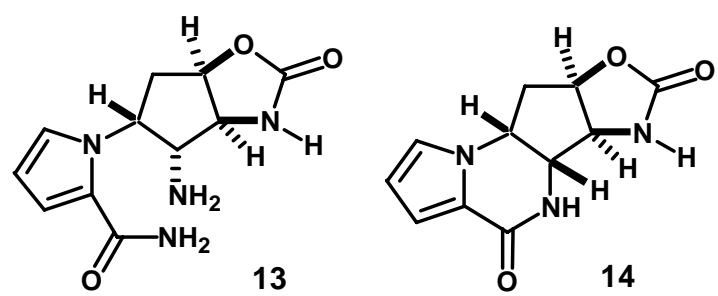

$(1 R, 9 S, 10 S, 14 R)-13-O x a-2,8,11-$ triazatetracyclo[7.6.0.0 $\left.0^{2,6} \cdot 0^{10,14}\right]$ pentadeca-3,5-diene-7,12-dione (14)

To a stirred solution of amide SI-2 (69 mg, $0.25 \mathrm{mmol})$ in $\mathrm{MeOH}(3 \mathrm{~mL})$ was added $\mathrm{Pd}(\mathrm{OH})_{2}$-C (20\% on carbon; $15 \mathrm{mg}$ ) at room temperature. After $35 \mathrm{~min}$ of stirring under hydrogen atmosphere, the mixture was filtered through Celite pad. The filtrate was concentrated under reduced pressure to give amine 13 (63 $\mathrm{mg}$ ) as a colorless oil. This material was subjected to the next lactamization reaction without purification. Thus, amine 13 (63 mg) was dissolved in $\mathrm{MeOH}(5 \mathrm{~mL})$, and then conc. $\mathrm{HCl}$ $(0.25 \mathrm{~mL})$ was added to this solution at room temperature. The mixture was heated at $60^{\circ} \mathrm{C}$ for $35 \mathrm{~min}$. NOTE: lactam 14 was extremely insoluble in many organic solvents. We found that DMF was a suitable solvent for dissolving this compound, enabling the efficient transfer of the solidified lactam 14 remained in the reaction vessel into flasks. After removal of the solvent under reduced pressure, the residual lactam 14 was rinsed with $\mathrm{MeOH}$. Lactam 14 obtained by this protocol was sufficiently pure (54.4 mg, 93\% in 2 steps). Amine 13: Colorless oil; IR (neat) v 3335, 2922, 1738, $1651 \mathrm{~cm}^{-1}$; ${ }^{1} \mathrm{H}$ NMR (300 MHz, CD $\left.{ }_{3} \mathrm{OD}\right) \delta 7.11$ (dd, 1H, $J=2.7,1.6 \mathrm{~Hz}$ ), 6.90 (dd, 1H, J=3.9, $1.6 \mathrm{~Hz}$ ), 6.16 (dd, $1 \mathrm{H}, J=3.9$, 
$2.7 \mathrm{~Hz}$ ), 5.84 (ddd, 1H, $J=13.6,6.2,5.1 \mathrm{~Hz}$ ), 5.19 (dd, 1H, $J=7.7,5.7 \mathrm{~Hz}$ ), 4.02 (d, 1H, $J=7.7 \mathrm{~Hz}$ ), 3.54 (d, 1H, J=5.1 Hz), 2.75 (td, 1H, J=13.6, $5.7 \mathrm{~Hz}), 2.30$ (dd, 1H, J=13.6, $6.2 \mathrm{~Hz}) ;{ }^{13} \mathrm{C}$ NMR $(75 \mathrm{MHz}$, $\left.\mathrm{CD}_{3} \mathrm{OD}\right) \delta 166.4,161.5,126.4,126.0,116.4,108.8,79.9,63.4,60.6,58.9,34.8$; MS m/z: $250\left(\mathrm{M}^{+}\right)$, 133 (100\%); HRMS (EI) calcd for $\mathrm{C}_{11} \mathrm{H}_{14} \mathrm{~N}_{4} \mathrm{O}_{3}\left(\mathrm{M}^{+}\right)$: 250.1066, found: 250.1045. Lactam 14: Colorless solid of mp $>300{ }^{\circ} \mathrm{C}$; IR $(\mathrm{KBr}) \vee$ 3219, 2907, 1736, $1647 \mathrm{~cm}^{-1}$; ${ }^{1} \mathrm{H}$ NMR $(500 \mathrm{MHz}$, DMSO- $d_{6}$ ) $\delta 8.05$ (brs, 1H), 7.68 (brs, 1H), 7.13 (dd, $1 \mathrm{H}, J=2.4,1.6 \mathrm{~Hz}$ ), 6.65 (dd, 1H, $J=3.8,1.6 \mathrm{~Hz}$ ), 6.14 (dd, 1H, J=3.8, 2.4 Hz), 5.14 (dd, 1H, $J=7.4,6.8 \mathrm{~Hz}$ ), 4.95 (ddd, 1H, $J=11.2,6.0,5.3 \mathrm{~Hz}$ ), 4.22 (d, 1H, J=7.4 Hz), 4.00 (d, 1H, J=5.3 Hz), 2.37 (dd, 1H, J=14.1, 6.8 Hz), 2.05 (ddd, 1H, J=14.1, 11.2, 6.0 $\mathrm{Hz}) ;{ }^{13} \mathrm{C}$ NMR (75 MHz, DMSO- $\left.d_{6}\right) \delta$ 158.6, 158.0, 124.1, 122.0, 112.6, 109.2, 78.2, 60.3, 60.0, 53.6, 38.7; MS m/z: $233\left(\mathrm{M}^{+}\right)$, 134 (100\%); HRMS (EI) calcd for $\mathrm{C}_{11} \mathrm{H}_{11} \mathrm{~N}_{3} \mathrm{O}_{3}\left(\mathrm{M}^{+}\right)$: 233.0800, found: 233.0807.

Urea 16 was synthesized by both route a and route $b$ whose details are given below. Route a: Three-step synthesis of 16 from 14<smiles>O=C1N[C@@H]2NC(=O)[C@H]3Cc4cccn4[C@@H]3C[C@H]2O1</smiles>

14<smiles>C[C@@]12NC(=O)c3cccn3[C@H]1C[C@@H](O)[C@@H]2NC(N)=O</smiles>

16

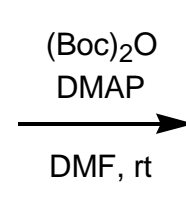

aq. $\mathrm{NaOH}$

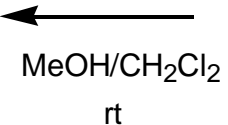

rt

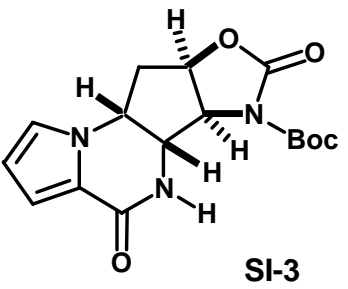

aq. $\mathrm{MeNH}_{2}$ $\mathrm{MeOH} / \mathrm{CH}_{2} \mathrm{Cl}_{2}$, rt

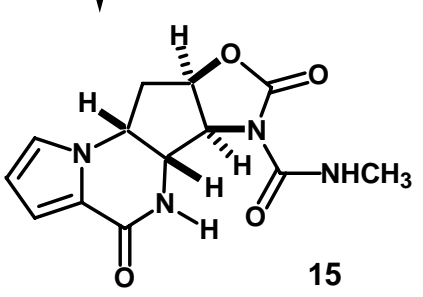

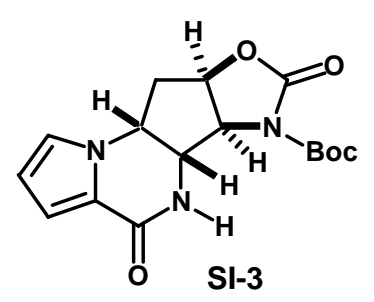

tert-Butyl(1R,9S,10S,14R)-7,12-dioxo-13-oxa-2,8,11-triazatetracyclo[7.6.0.0 $\left.{ }^{2,6} \cdot 0^{10,14}\right]$ pentadeca-3,5 -diene-11-carboxylate (SI-3) 
To a stirred solution of lactam 14 (45 mg, $0.194 \mathrm{mmol})$ in DMF (4 mL) were added $\mathrm{Boc}_{2} \mathrm{O}(89 \mu \mathrm{L}$, $0.39 \mathrm{mmol}$ ) and DMAP (12 mg, $0.1 \mathrm{mmol})$ at room temperature. After $10 \mathrm{~min}$, sat. $\mathrm{NH}_{4} \mathrm{Cl}$ was added to the solution, and the whole mixture was then extracted with EtOAc. The organic extracts were combined, dried over $\mathrm{MgSO}_{4}$, filtered, and concentrated to give Boc derivative SI-3 (60.1 mg, 93\%) as a colorless solid. This sufficiently pure material was used for the next reaction without purification. Colorless cubes of $\mathrm{mp}>300{ }^{\circ} \mathrm{C}$; $[\alpha]_{\mathrm{D}}{ }^{26}-12.7\left(c 0.71, \mathrm{CHCl}_{3}\right)$; IR $(\mathrm{KBr}) \vee 3331,2932,1792,1661 \mathrm{~cm}^{-1}$; ${ }^{1} \mathrm{H}$ NMR (300 MHz, $\mathrm{CDCl}_{3}$ ) $\delta 6.99$ (dd, 1H, J=3.6, $1.5 \mathrm{~Hz}$ ), 6.86 (dd, 1H, J=2.5, $1.5 \mathrm{~Hz}$ ), 6.52 (brs, 1H), 6.30 (dd, 1H, J=3.6, $2.5 \mathrm{~Hz}$ ), 5.06 (ddd, 1H, $J=7.7,5.4,1.5 \mathrm{~Hz}$ ), 4.79 (ddd, 1H, $J=9.7,6.5,5.6$ Hz), 4.57 (d, 1H, J=7.7 Hz), 4.36 (d, 1H, J=5.6 Hz), 2.57 (ddd, 1H, J=14.8, 6.5, 1.5 Hz), 2.50 (ddd, 1H, $J=14.8,9.7,5.4 \mathrm{~Hz}$ ), 1.57 (s, 9H); ${ }^{13} \mathrm{C}$ NMR (75 MHz, DMSO-d $\left.{ }_{6}\right) \delta 158.4,151.2,148.4,123.8$, 122.1, 112.6, 109.3, 83.1, 75.6, 62.9, 59.1, 53.4, 37.7, 27.6; MS m/z: $333\left(\mathrm{M}^{+}\right), 56$ (100\%); HRMS (EI) calcd for $\mathrm{C}_{16} \mathrm{H}_{19} \mathrm{~N}_{3} \mathrm{O}_{5}\left(\mathrm{M}^{+}\right)$: 333.1324, found: 333.1336.

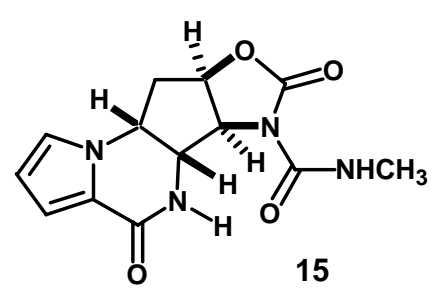

$(1 R, 9 S, 10 S, 14 R)-N$-Methyl-7,12-dioxo-13-oxa-2,8,11-triazatetracyclo[7.6.0.0 $\left.{ }^{2,6} \cdot 0^{10,14}\right]$ pentadeca-3,

\section{5-diene-11-carboxamide (15)}

To a solution of Boc derivative SI-3 (23.5 mg, $0.071 \mathrm{mmol})$ in $\mathrm{MeOH}-\mathrm{CH}_{2} \mathrm{Cl}_{2}(1: 1 \mathrm{v} / \mathrm{v} 8 \mathrm{~mL})$ was added $40 \%$ aq. $\mathrm{MeNH}_{2}(0.5 \mathrm{~mL}, 5.78 \mathrm{mmol})$ at room temperature. The reaction mixture was stirred at the same temperature for $100 \mathrm{~min}$ and then concentrated under reduced pressure. The residue was diluted with EtOAc and transferred to a separatory funnel where it was washed with water. The organic extracts were combined, dried over $\mathrm{MgSO}_{4}$, filtered, and concentrated. The residue was purified by flash silica gel column chromatography (EtOAc/hexane 2:1) to give urea 15 (14.9 mg, $73 \%)$ as a colorless oil. Colorless oil; $[\alpha]_{\mathrm{D}}{ }^{26}-40.0\left(c\right.$ 0.41, $\left.\mathrm{CHCl}_{3}\right)$; IR (neat) $v 3354,2926,1759,1659$, $1651 \mathrm{~cm}^{-1} ;{ }^{1} \mathrm{H}$ NMR (300 MHz, $\mathrm{CDCl}_{3}$ ) $\delta 7.65$ (brs, $1 \mathrm{H}$ ), 7.00 (dd, 1H, J=3.8, 1.6 Hz), 6.82 (dd, 1H, $J=2.6,1.6 \mathrm{~Hz}$ ), 6.30 (dd, 1H, J=3.8, 2.6 Hz), 6.06 (brs, 1H), 5.09 (ddd, 1H, J=8.4, 5.7, 3.1 Hz), 4.75 (dt, 1H, $J=8.1,5.8 \mathrm{~Hz}$ ), 4.61 (dd, 1H, $J=8.1,1.8 \mathrm{~Hz}$ ), 4.37 (m, 1H), 2.91 (d, 3H, $J=4.8 \mathrm{~Hz}$ ), 2.66 (ddd, 
1H, $J=14.7,8.4,5.8 \mathrm{~Hz}$ ), 2.51 (ddd, $1 \mathrm{H}, J=14.7,5.8,3.1 \mathrm{~Hz}$ ); ${ }^{13} \mathrm{C}$ NMR $\left(125 \mathrm{MHz}, \mathrm{CDCl}_{3}\right) \delta 159.2$, 154.5, 151.8, 122.7, 122.4, 115.2, 111.2, 76.7, 64.2, 60.8, 54.3, 38.3, 26.7; MS m/z: $290\left(\mathrm{M}^{+}\right), 172$ (100\%); HRMS (EI) calcd for $\mathrm{C}_{13} \mathrm{H}_{14} \mathrm{~N}_{4} \mathrm{O}_{4}\left(\mathrm{M}^{+}\right)$: 290.1015, found: 290.1008 .

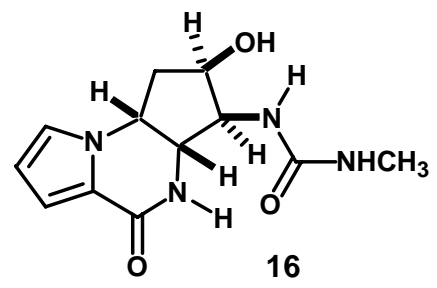

\section{1-[(2R,4R,5S,6S)-4-Hydroxy-8-oxo-1,7-diazatricyclo[7.3.0.0 $\left.{ }^{2,6}\right]$ dodeca-9,11-dien-5-yl]-3-methylur} ea (16):

To a stirred solution of urea 15 (50.5 mg, $0.174 \mathrm{mmol})$ in $\mathrm{MeOH}-\mathrm{CH}_{2} \mathrm{Cl}_{2}(3: 2 \mathrm{v} / \mathrm{v} 5 \mathrm{~mL})$ was added $1 \mathrm{~N}$ $\mathrm{NaOH}(0.5 \mathrm{~mL})$ at room temperature. After $1.5 \mathrm{~h}$, additional $1 \mathrm{~N} \mathrm{NaOH}(0.5 \mathrm{~mL})$ was added and stirring was continued for further $2 \mathrm{~h}$. The mixture was neutralized with $1 \mathrm{~N} \mathrm{HCl}$ and concentrated under reduced pressure. The residue was purified by flash silica gel column chromatography (MeOH /EtOAc 1:5 $\rightarrow 1: 3$ ) to give alcohol $\mathbf{1 6}$ (36.8 $\mathrm{mg}, \mathbf{8 0 \% )}$ as a colorless solid. Colorless needles of $\mathrm{mp}$ 240-241 ${ }^{\circ} \mathrm{C}(\mathrm{MeOH}) ;[\alpha]_{\mathrm{D}}{ }^{27}-74.6$ (c 0.14, MeOH); IR (KBr) v 3397, 2926, $1634 \mathrm{~cm}^{-1} ;{ }^{1} \mathrm{H}$ NMR (300 $\mathrm{MHz}, \mathrm{CD}_{3} \mathrm{OD}$ ) $\delta 7.01$ (dd, 1H, J=2.6, $1.6 \mathrm{~Hz}$ ), 6.85 (dd, 1H, $J=3.8,1.6 \mathrm{~Hz}$ ), 6.27 (dd, 1H, $J=3.8,2.6$ Hz), 4.70 (td, 1H, J=7.1, 4.8 Hz), 4.17 (ddd, 1H, J=6.0, 4.6, 2.6 Hz), 3.97 (m, 2H), 2.69 (s, 3H), 2.53 (ddd, 1H, $J=14.9,6.0,4.8 \mathrm{~Hz}$ ), 2.39 (ddd, 1H, $J=14.9,7.1,2.6 \mathrm{~Hz}) ;{ }^{13} \mathrm{C}$ NMR (125 MHz, DMSO-d 6 ) $\delta$ 158.8, 157.7, 123.3, 122.4, 111.8, 109.5, 67.4, 58.8, 57.2, 51.3, 39.7, 26.3; MS m/z: $264\left(\mathrm{M}^{+}\right), 233$ (100\%); HRMS (EI) calcd for $\mathrm{C}_{12} \mathrm{H}_{16} \mathrm{~N}_{4} \mathrm{O}_{3}\left(\mathrm{M}^{+}\right)$: 264.1222, found: 264.1232 .

Route b: Direct synthesis of urea 16 by aminolysis of oxazolidinone 14 with $\mathrm{MeNH}_{2}$
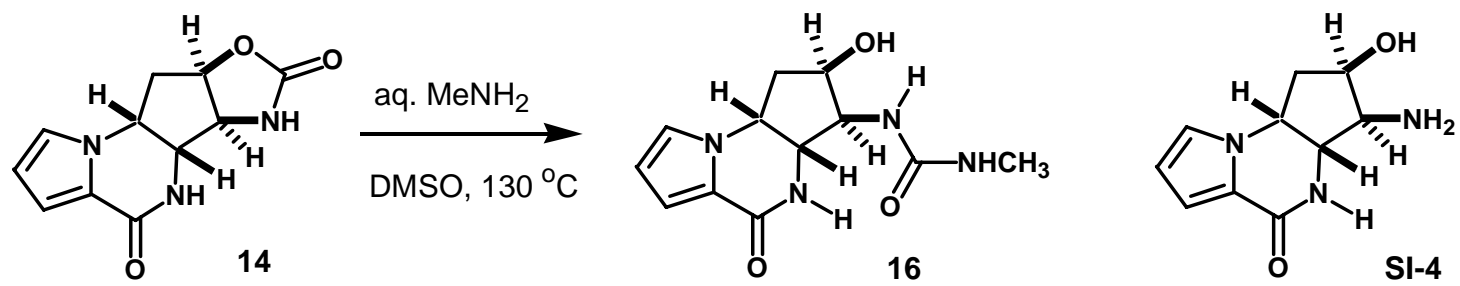

To a solution of lactam $14(10.8 \mathrm{mg}, 0.046 \mathrm{mmol})$ in DMSO $(1 \mathrm{~mL})$ in a high-pressure stainless steel tube was added $40 \%$ aq. $\mathrm{MeNH}_{2}(1 \mathrm{~mL}, 11.5 \mathrm{mmol})$ at room temperature, and the mixture was heated at $130^{\circ} \mathrm{C}$ for $3.5 \mathrm{~h}$. Additional $40 \%$ aq. $\mathrm{MeNH}_{2}(1 \mathrm{~mL}, 11.5 \mathrm{mmol})$ was added and heating was 
continued at $130^{\circ} \mathrm{C}$ for further $5 \mathrm{~h}$. After concentration of the mixture under reduced pressure, the residual lactam 14 was rinsed with $\mathrm{MeOH}$ to leave sufficiently pure urea 16 (8.3 mg, 68\%) as a colorless solid. Concentration of the $\mathrm{MeOH}$ extracts under reduced pressure afforded aminoalcohol SI-4 (2.8 mg, 30\%) as a colorless solid. Aminoalcohol SI-4: Colorless solid; IR (neat) v 3335, 2938, $1645 \mathrm{~cm}^{-1}$; ${ }^{1} \mathrm{H}-\mathrm{NMR}\left(500 \mathrm{MHz}, \mathrm{CD}_{3} \mathrm{OD}\right) \delta 7.01$ (dd, $1 \mathrm{H}, J=2.6,1.6 \mathrm{~Hz}$ ), 6.86 (dd, $1 \mathrm{H}, J=3.8,1.6 \mathrm{~Hz}$ ), 6.27 (dd, 1H, $J=3.8,2.6 \mathrm{~Hz}$ ), 4.72 (ddd, 1H, $J=7.3,7.1,3.8 \mathrm{~Hz}$ ), 4.18 (ddd, 1H, $J=5.9,5.5,2.7 \mathrm{~Hz}$ ), 3.95 (dd, 1H, J=8.8, 7.1 Hz), 3.15 (dd, 1H, J=8.8, $5.5 \mathrm{~Hz}$ ), 2.57 (ddd, 1H, $J=15.1,5.9,3.8 \mathrm{~Hz}$ ), 2.45 (ddd, 1H, $J=15.1,7.3,2.7 \mathrm{~Hz}$ ).

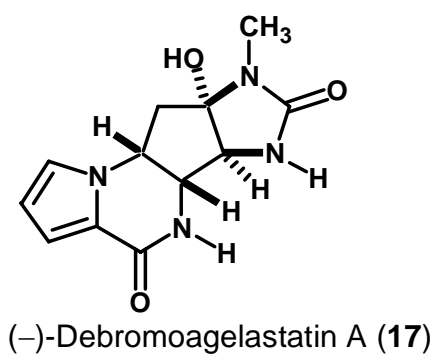

\section{(-)-Debromoagelastatin A (17)}

To a stirred solution of alcohol 16 (25.9 $\mathrm{mg}, 0.098 \mathrm{mmol})$ in DMF (4 mL) were added a solution of NMO (17.2 mg, $0.147 \mathrm{mmol})$ in DMF (1 mL) and a solution of TPAP (17.2 mg, $0.049 \mathrm{mmol})$ in DMF $(1 \mathrm{~mL})$ at room temperature. After being stirred for $1 \mathrm{~h}$, the mixture was treated with $i$-PrOH $(200 \mu \mathrm{L})$ followed by sat. $\mathrm{NH}_{4} \mathrm{Cl}(200 \mu \mathrm{L})$, and stirring was continued for an additional $5 \mathrm{~min}$. Then the mixture was concentrated under reduced pressure, and the residue was purified by flash silica gel column chromatography (MeOH/EtOAc 1:5 $\rightarrow 1: 3)$ to give (-)-debromoagelastatin A (17) (11.4 mg, 44\%) as a colorless solid. Colorless solid of mp $242-244^{\circ} \mathrm{C}$; $[\alpha]_{\mathrm{D}}{ }^{25}-68.2$ (c 0.14, MeOH); IR (KBr) v 3366, 2924, 1682, $1638 \mathrm{~cm}^{-1}$; ${ }^{1} \mathrm{H}$ NMR (500 MHz, $\mathrm{CD}_{3} \mathrm{OD}$ ) $\delta 7.02$ (dd, $1 \mathrm{H}, J=2.6,1.6 \mathrm{~Hz}$ ), 6.88 (dd, $1 \mathrm{H}, J=3.9$, 1.6 Hz), 6.23 (dd, 1H, J=3.9, 2.6 Hz), 4.65 (ddd, 1H, J=10.2, 6.4, $5.6 \mathrm{~Hz}$ ), 3.99 (dd, 1H, J=5.6, $1.2 \mathrm{~Hz}$ ), 3.80 (d, 1H, J=1.2 Hz), 2.79 (s, 3H), 2.62 (dd, 1H, $J=13.3,6.4 \mathrm{~Hz}$ ), 2.27 (dd, 1H, $J=13.3,10.2 \mathrm{~Hz}$ ); ${ }^{13} \mathrm{C}$ NMR (125 MHz, $\left.\mathrm{CD}_{3} \mathrm{OD}\right) \delta 162.1,161.3,125.6,122.9,115.4,111.1,95.8,68.0,62.9,55.6,24.2$; MS m/z: $263\left(\mathrm{MH}^{+}\right), 59$ (100\%); HRMS (FAB) calcd for $\mathrm{C}_{12} \mathrm{H}_{15} \mathrm{~N}_{4} \mathrm{O}_{3}\left(\mathrm{MH}^{+}\right)$: 263.1144, found: 263.1146. The spectroscopic and analytical data of this material were identical with those reported. 


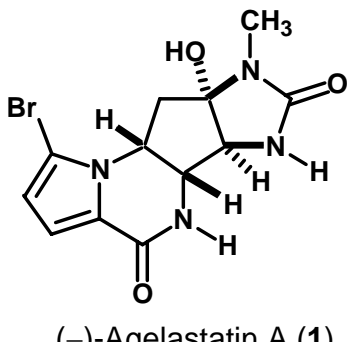

\section{(-)-Agelastatin A (1)}

To a solution of debromoagelastatin A (17) (10.1 mg, $0.039 \mathrm{mmol})$ in MeOH-THF (1:2 v/v $6 \mathrm{~mL})$ was added NBS (6.2 $\mathrm{mg}, 0.035 \mathrm{mmol}$ ) at $0^{\circ} \mathrm{C}$, and the mixture was allowed to warm to room temperature. After being stirred for $5 \mathrm{~min}$, the mixture was concentrated under reduced pressure. The residue was purified by flash silica gel column chromatography $\left(\mathrm{MeOH} / \mathrm{CH}_{2} \mathrm{Cl}_{2} 1: 9 \rightarrow 1: 5\right)$ to give (-)-agelastatin A (1) $(9.6 \mathrm{mg}, 73 \%)$ as a colorless solid. Colorless solid of mp $196^{\circ} \mathrm{C}$; $[\alpha]_{\mathrm{D}}{ }^{26}-64.4$ (c 0.15, MeOH) [lit. ${ }^{2 \mathrm{a}}$ $[\alpha]_{\mathrm{D}}{ }^{20}-65.5$ (c 0.5, MeOH); lit. $^{2 \mathrm{~b}}[\alpha]_{\mathrm{D}}-84.2$ (c 1.0, MeOH); lit. $^{2 \mathrm{c}}[\alpha]_{\mathrm{D}}{ }^{20}-62.2$ (c 0.18, MeOH); lit. ${ }^{2 \mathrm{~d}}$ $[\alpha]_{\mathrm{D}}{ }^{14}-83.8$ (c 0.21, MeOH)]; IR (KBr) v 3279, 2926, $1641 \mathrm{~cm}^{-1}$; ${ }^{1} \mathrm{H}$ NMR (500 MHz, CD $\left.{ }_{3} \mathrm{OD}\right) \delta 6.90$ (d, 1H, J=4.1 Hz), 6.32 (d, 1H, J=4.1 Hz), 4.59 (ddd, 1H, J=12.0, 6.5, 5.5 Hz), 4.08 (d, 1H, J=5.5 Hz), 3.87 (s, 1H), 2.80 (s, 3H), 2.64 (dd, 1H, J=13.1, $6.5 \mathrm{~Hz}$ ), 2.09 (dd, $1 \mathrm{H}, J=13.1,12.0 \mathrm{~Hz}$ ); ${ }^{13} \mathrm{C}$ NMR (125 MHz, CD $\left.{ }_{3} \mathrm{OD}\right) \delta 161.4,161.1,124.2,116.0,113.8,107.2,95.7,67.4,62.2,54.4,40.0,24.2 ; \mathrm{MS}$ m/z: $341\left(\mathrm{MH}^{+}\right), 93$ (100\%); HRMS (FAB) calcd for $\mathrm{C}_{12} \mathrm{H}_{14} \mathrm{~N}_{4} \mathrm{O}_{3}{ }^{79} \mathrm{Br}\left(\mathrm{MH}^{+}\right)$: 341.0249, found: 341.0271. The spectroscopic and analytical data of this material were in good agreement with those reported.

\section{References}

1. (a) Davis, F. A.; Wu, Y. Org. Lett. 2004, 6, 1269. (b) Mulvihill, M. J.; Gage, J. L.; Miller, M. J. J. Org. Chem. 1998, 63, 3357.

2. (a) Feldman, K. S.; Saunders, J. C. J. Am. Chem. Soc. 2002, 124, 9060. (b) Hale, K. J.; Domostoj, M. M.; Tocher, D. A.; Irving, E.; Scheinmann, F. Org. Lett. 2003, 5, 2927. (c) Davis, F. A.; Deng, J. Org. Lett. 2005, 7, 621. (d) Ichikawa, Y.; Yamaoka, T.; Nakano, K.; Kotsuki, H. Org. Lett. 2007, 9, 2989. 
o" "I

$70 \cdot 0 I$ 


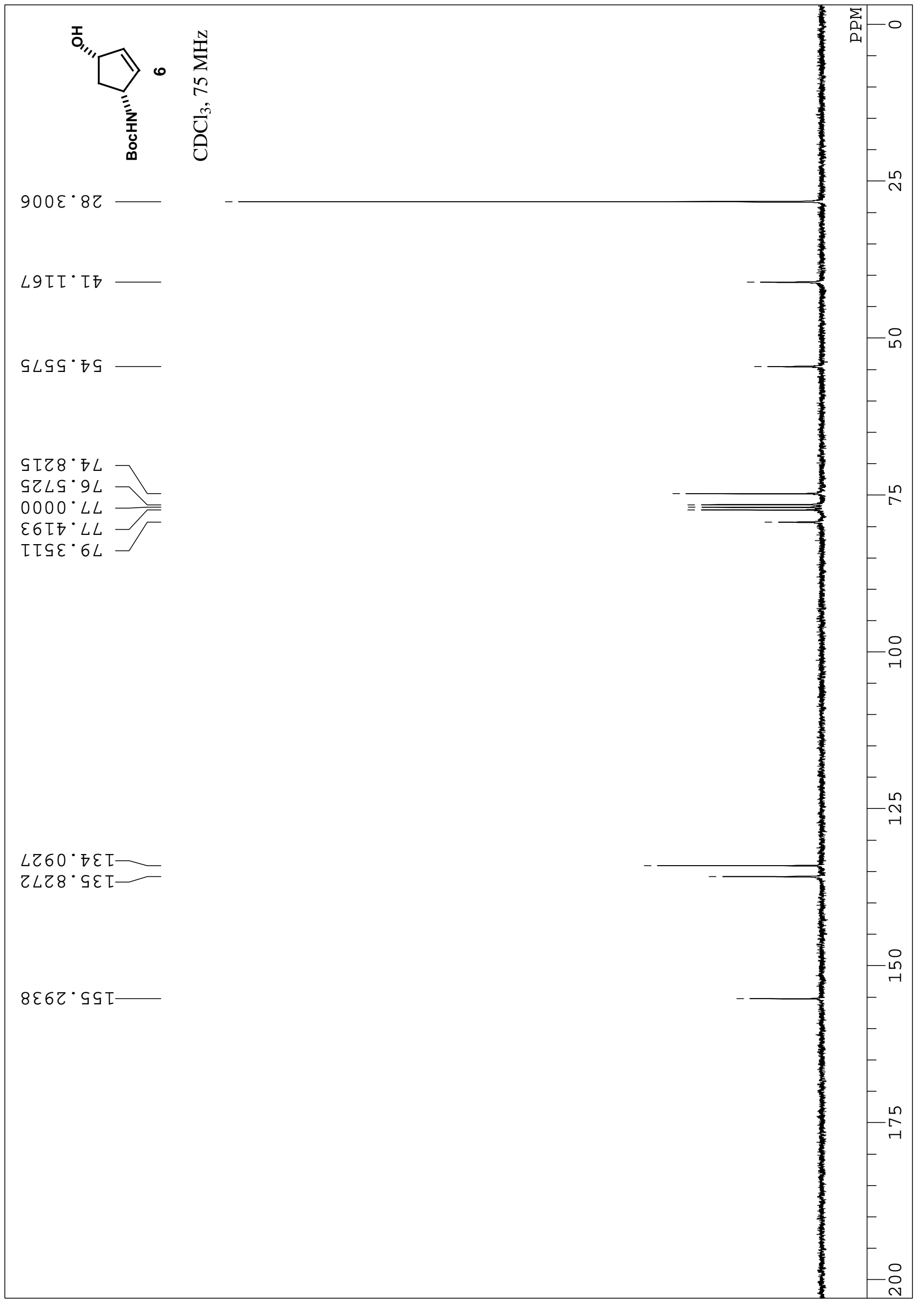




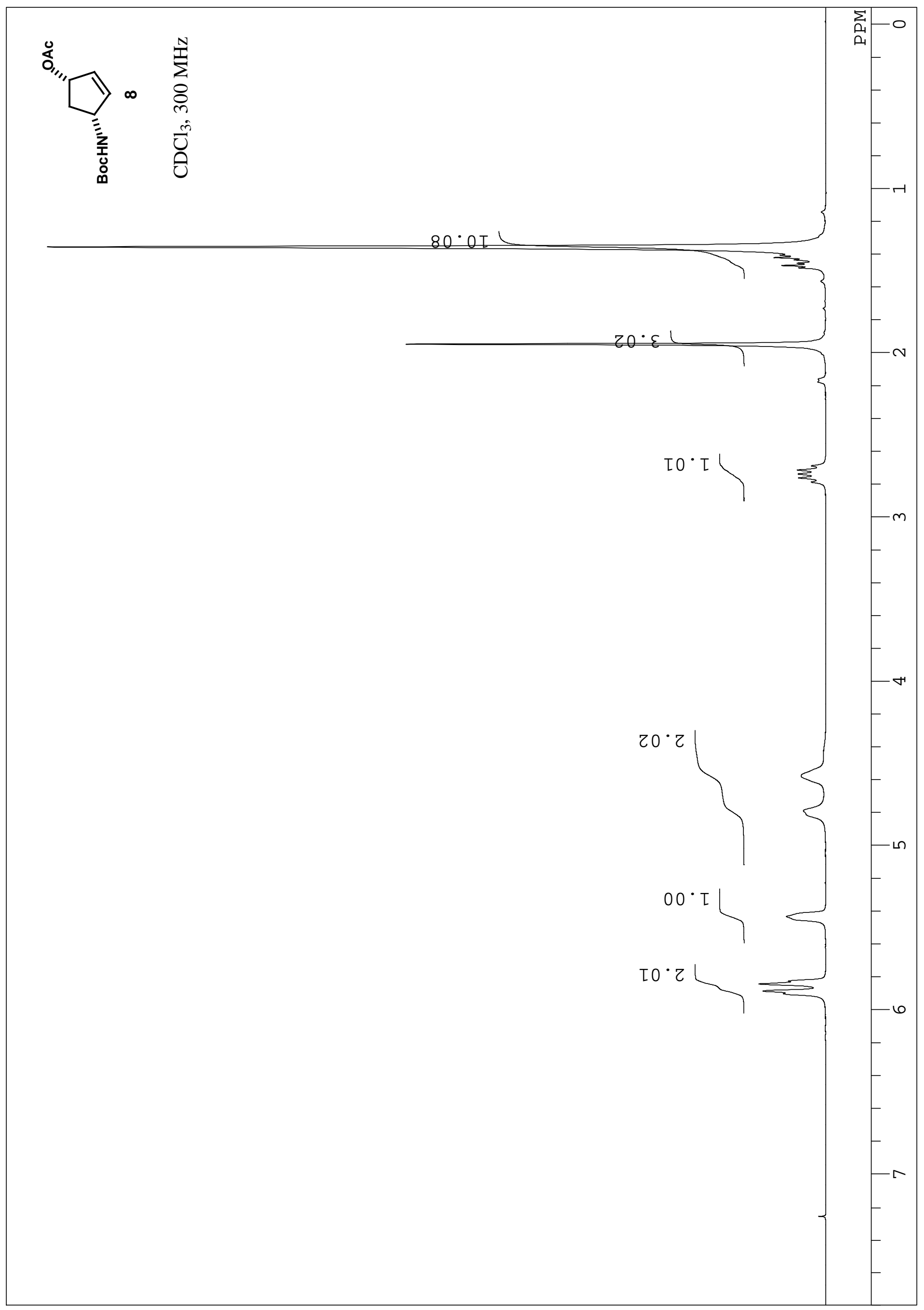




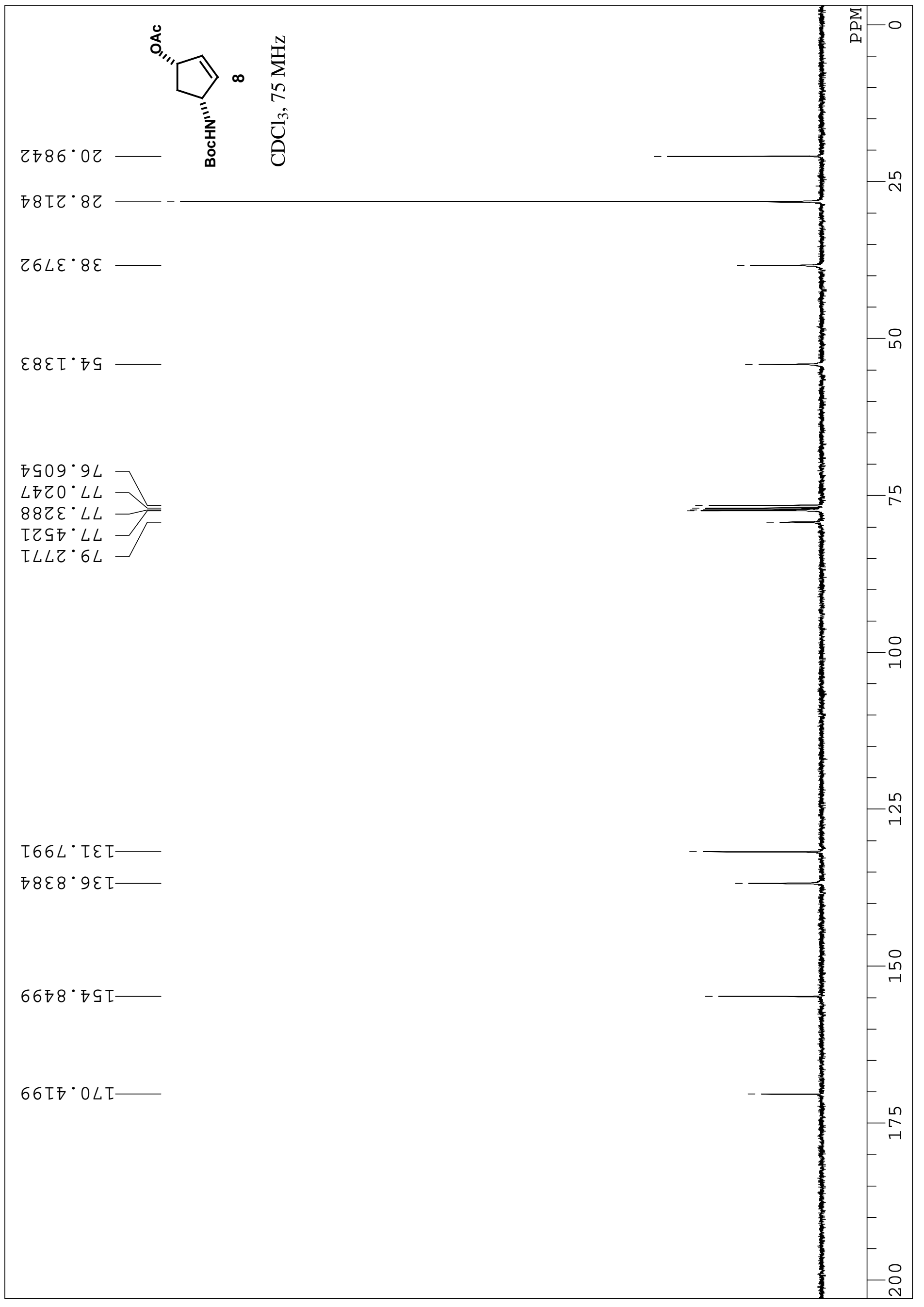




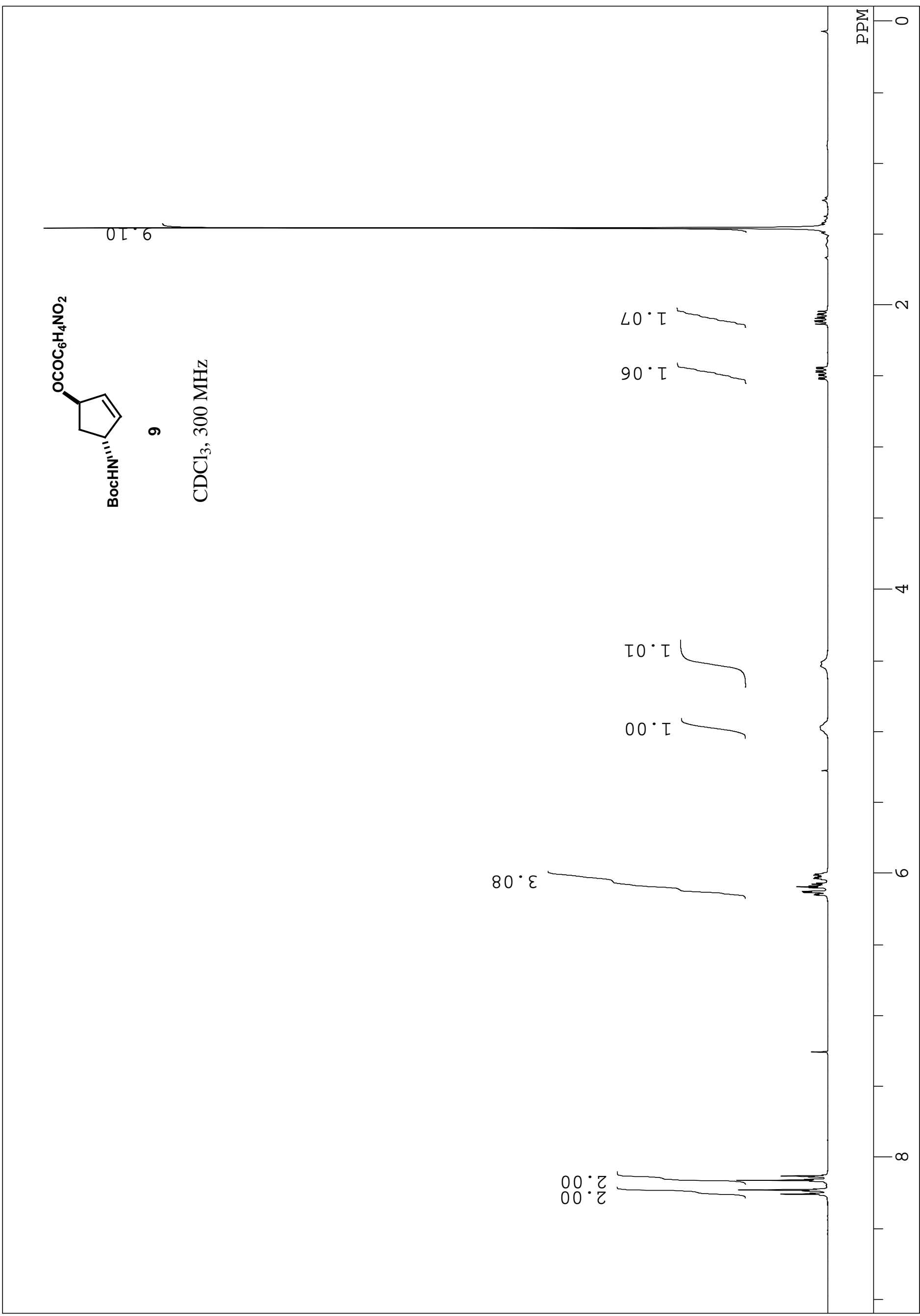




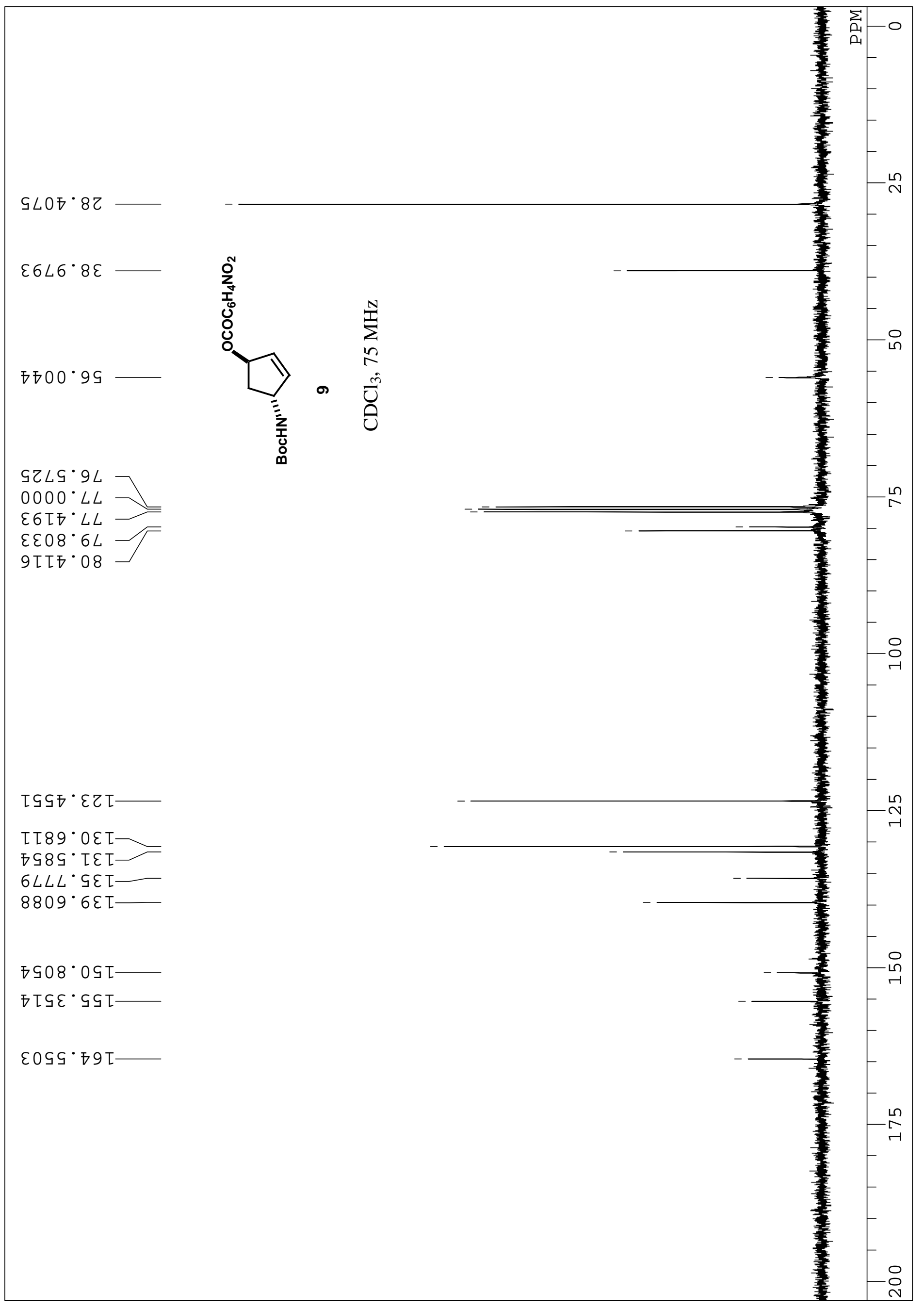



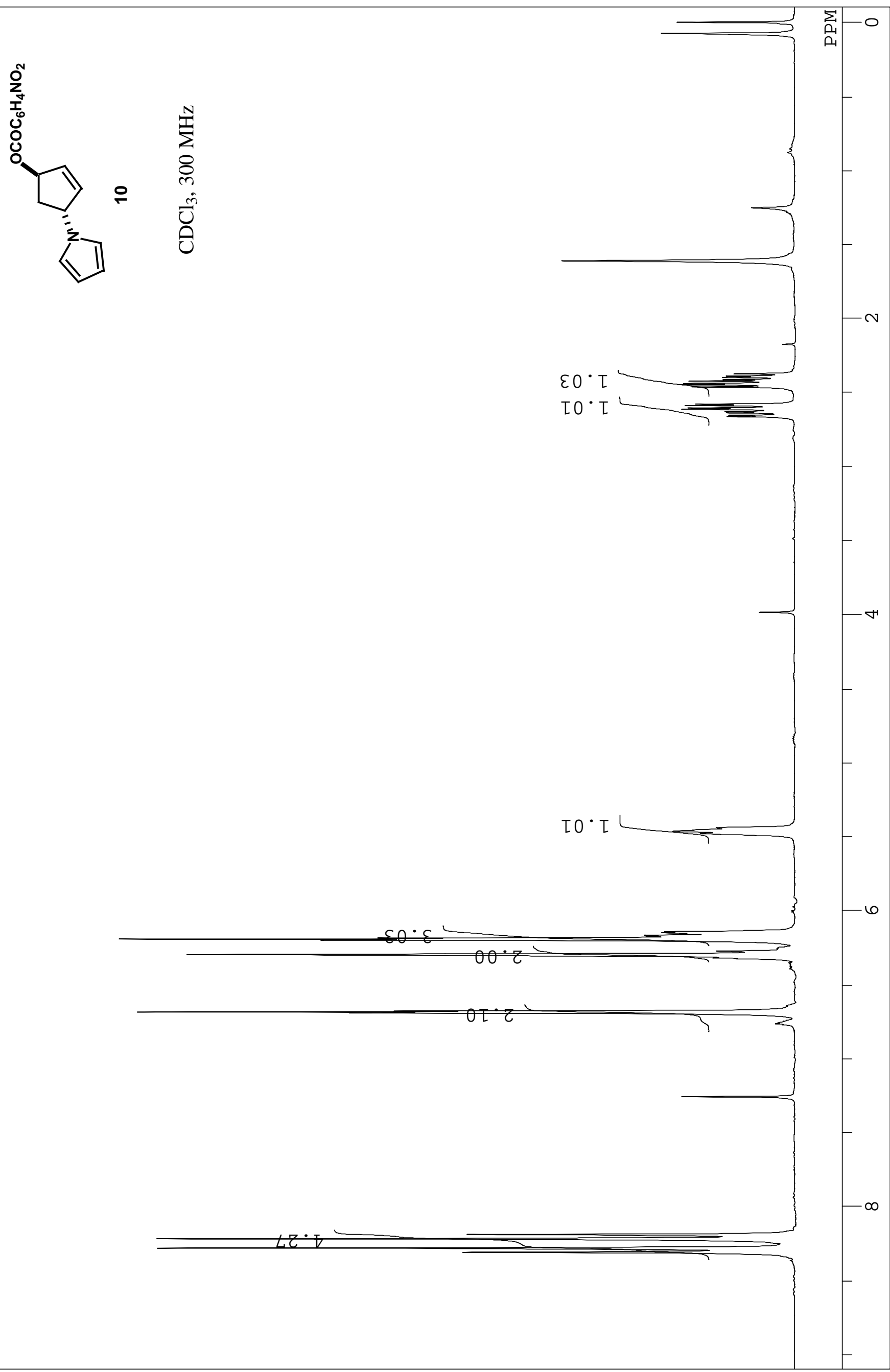


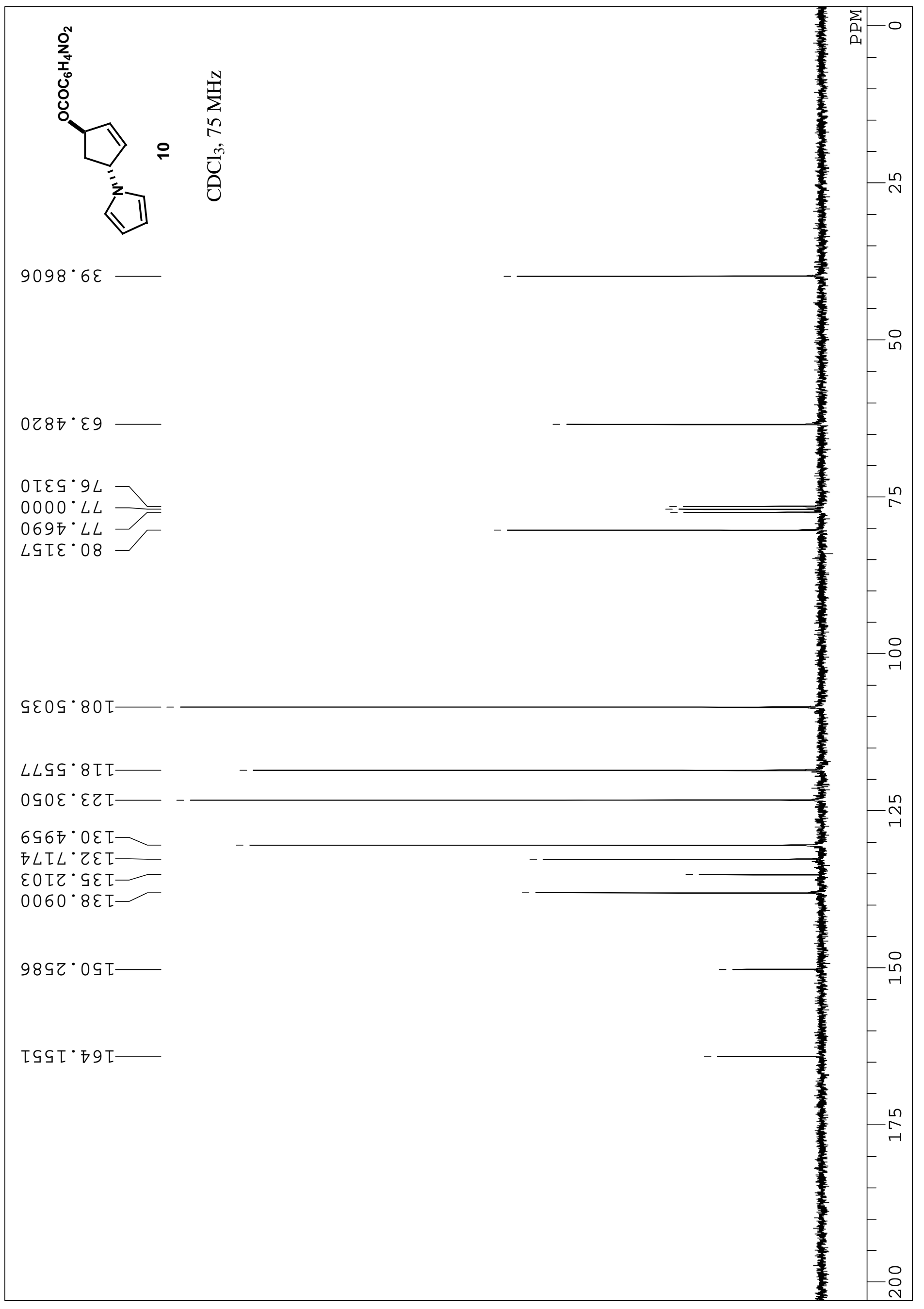



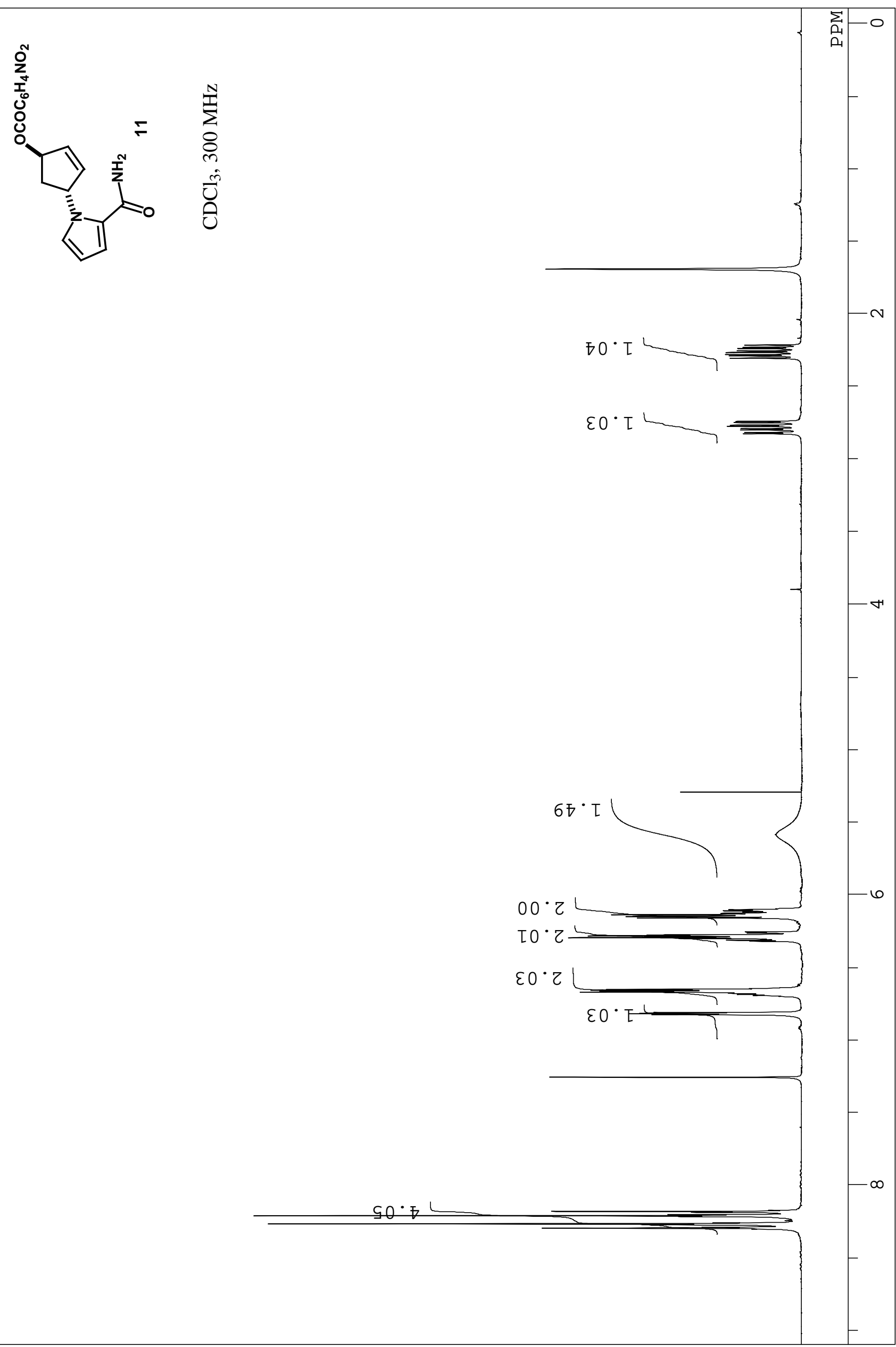


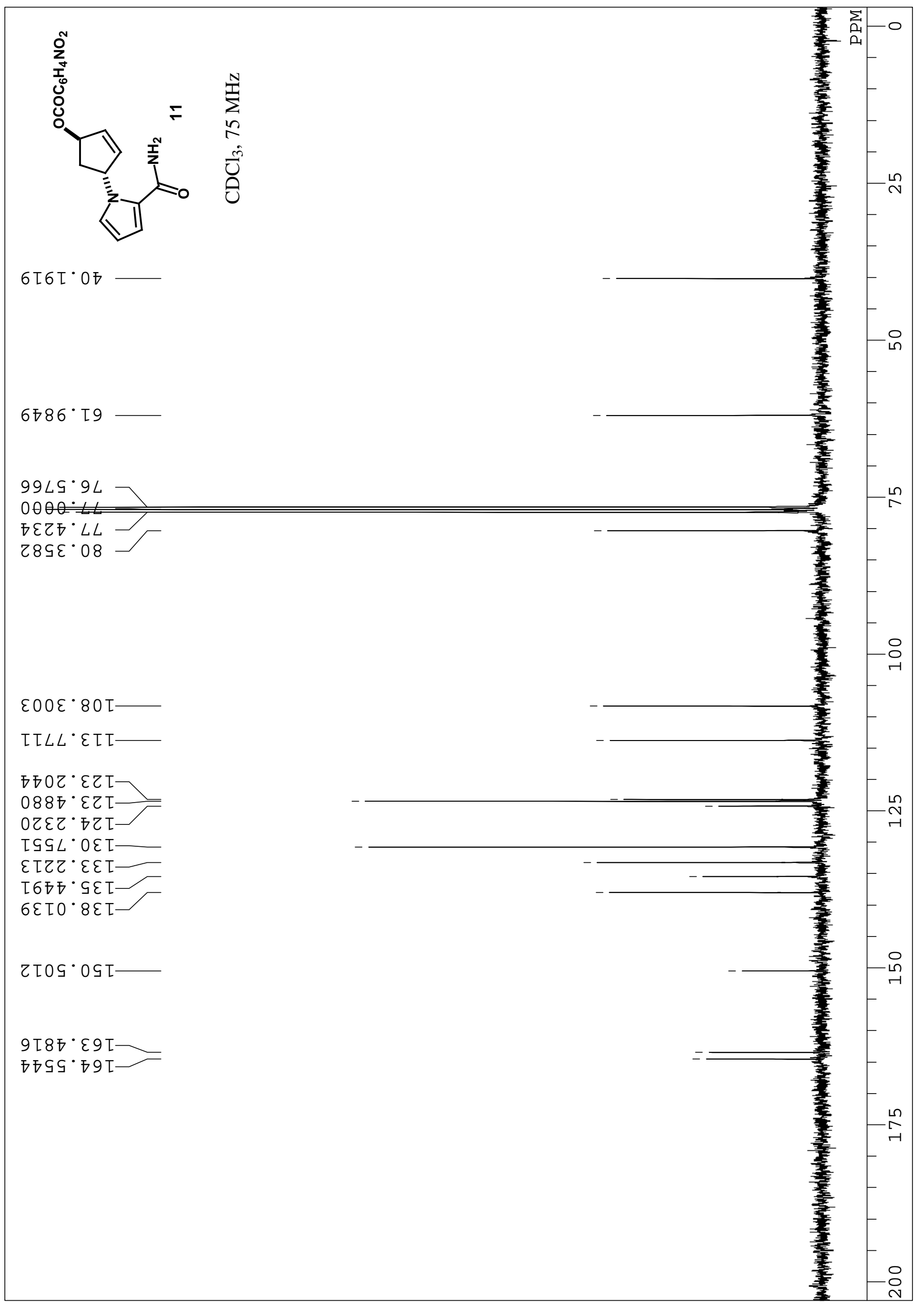



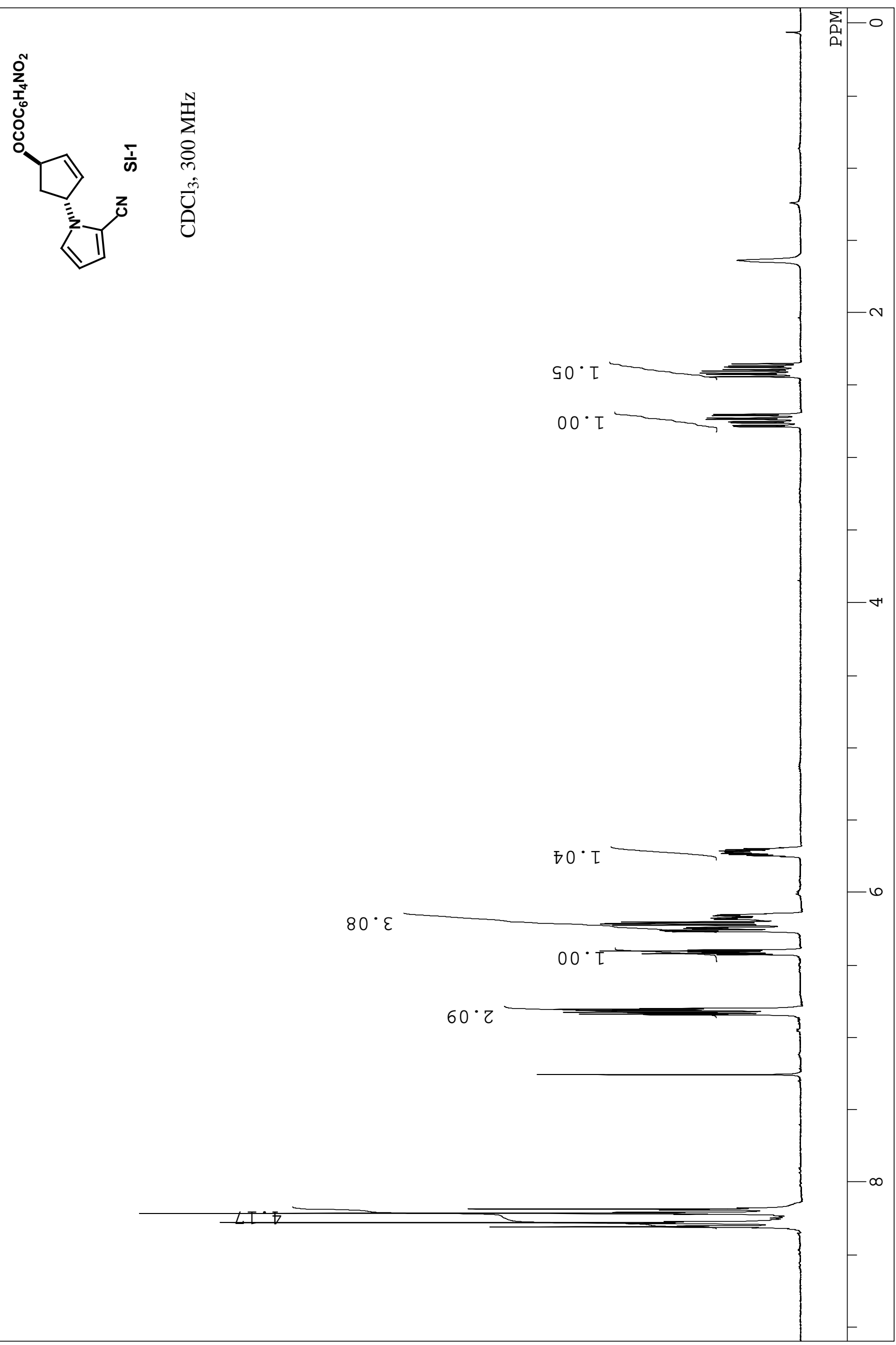


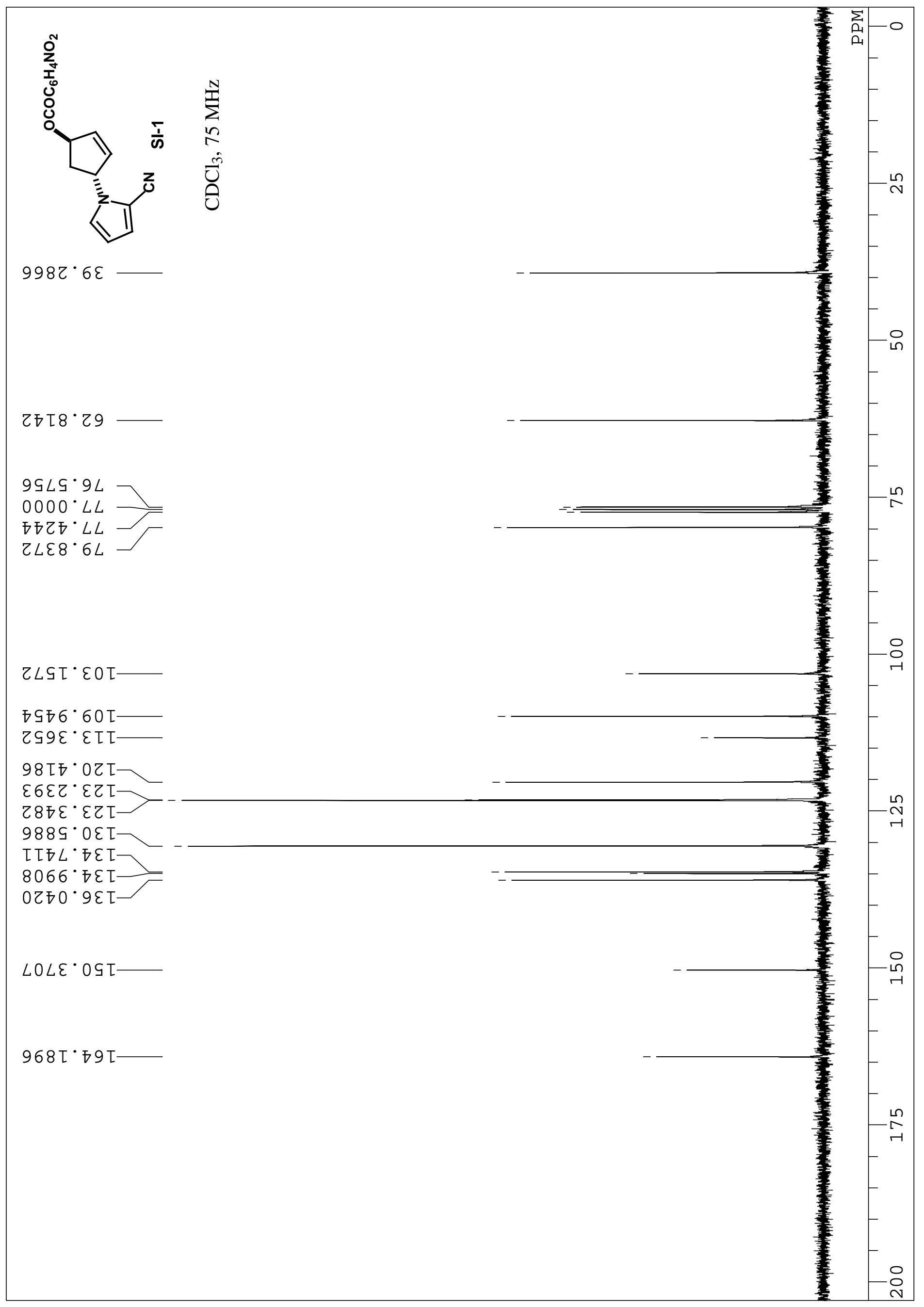


\11

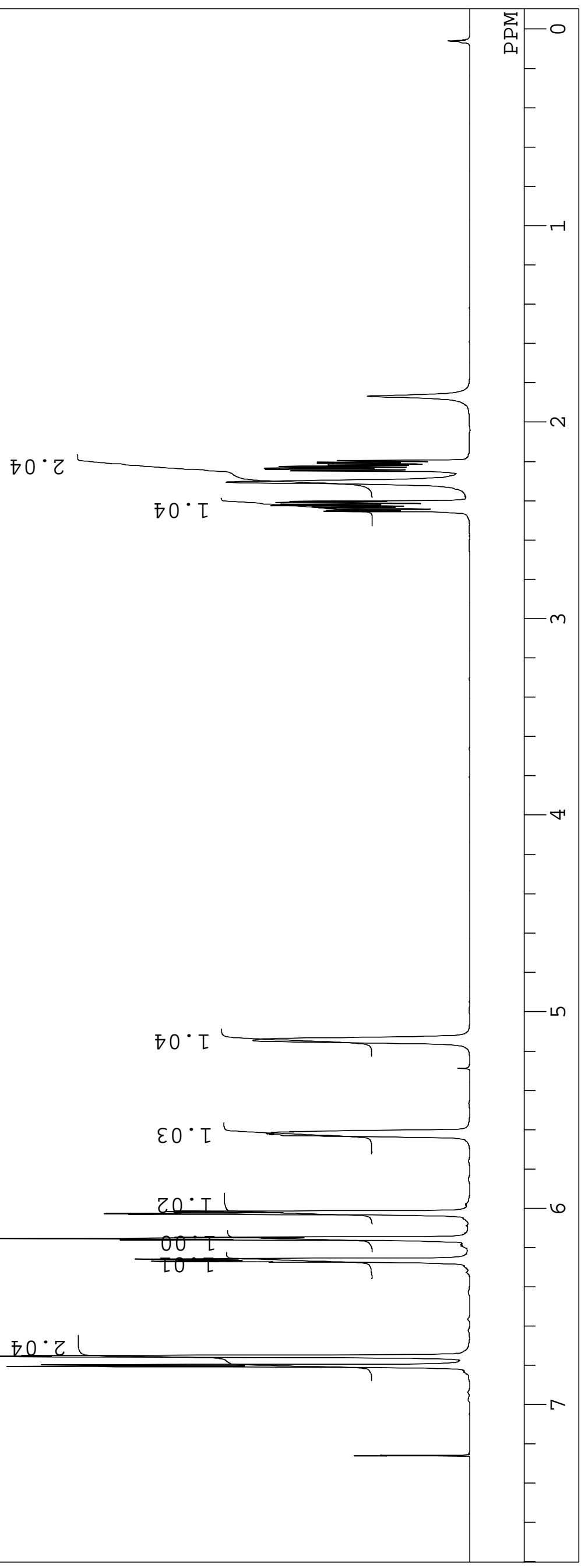




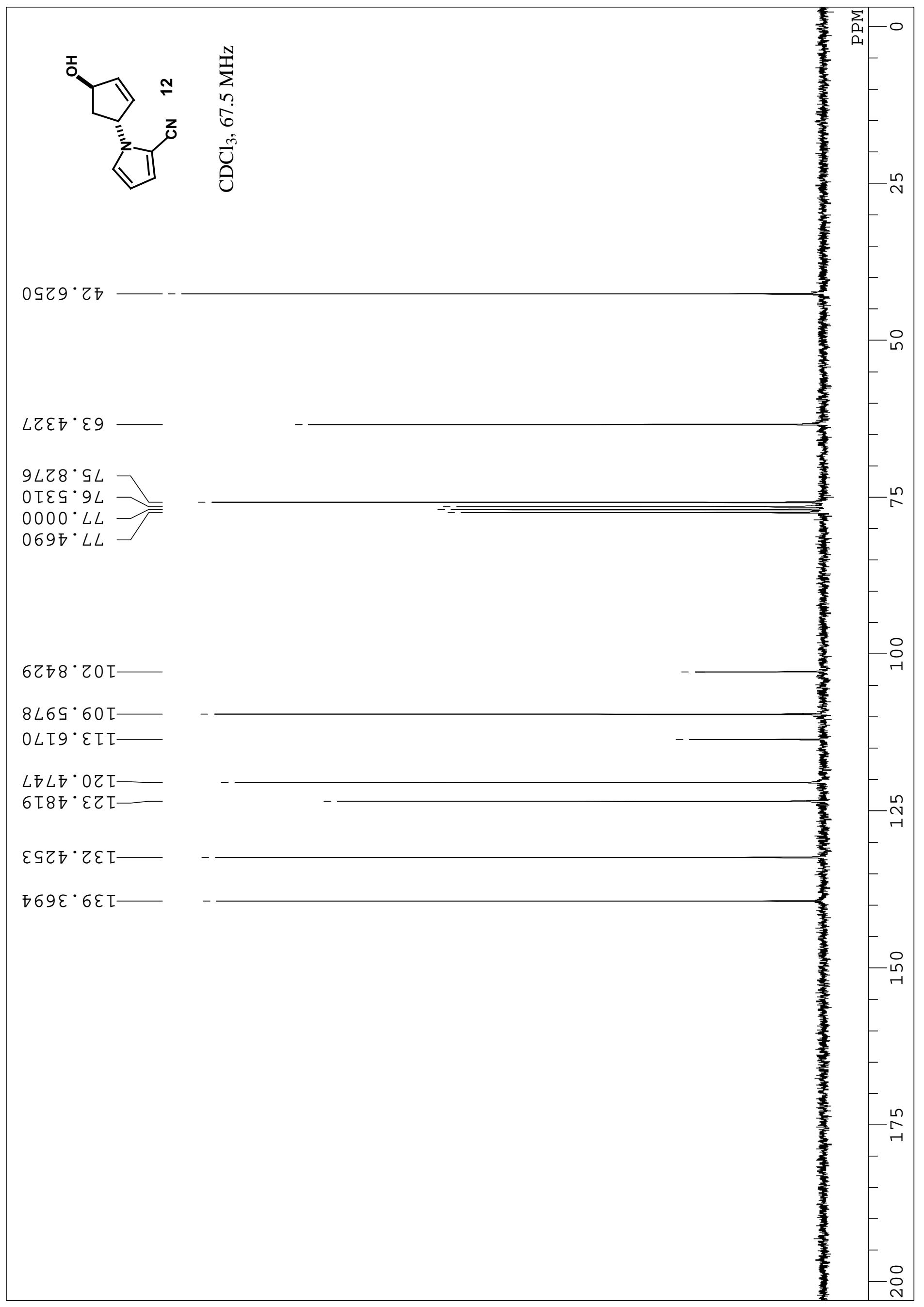


(1)

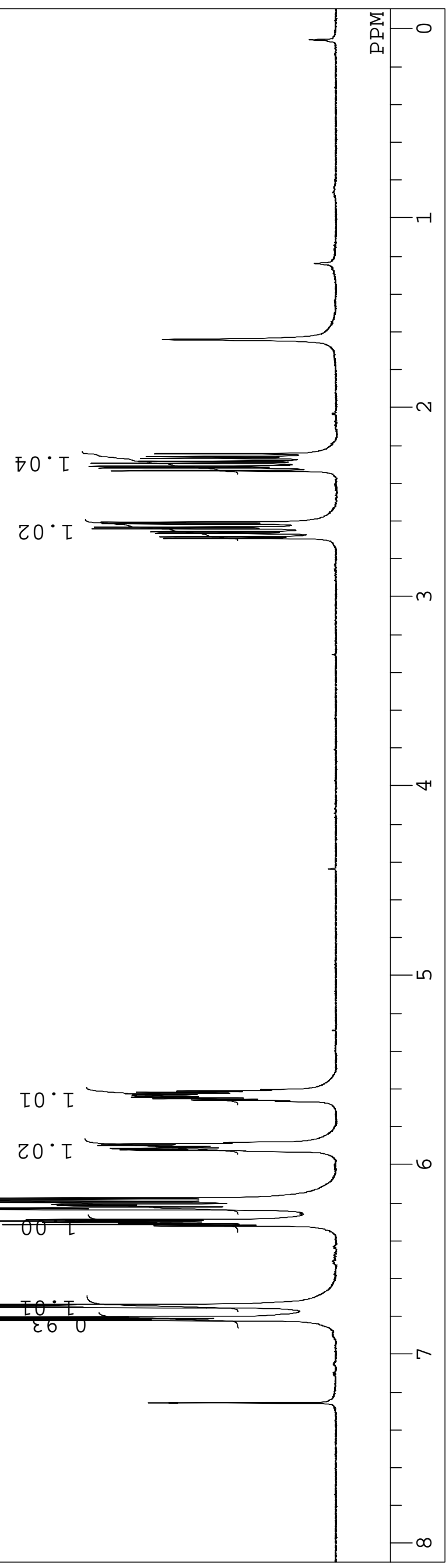




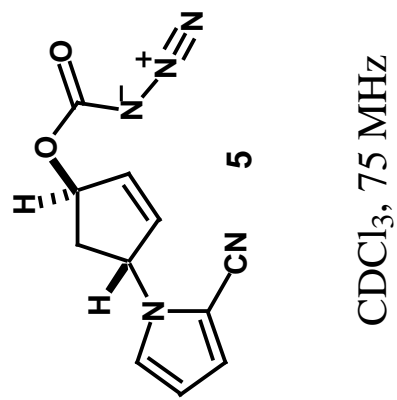

$2 L 6 \tau \cdot 6 \varepsilon$

$0 \nabla 78 \cdot 29$

$99 L G \cdot 9 L$ $0000^{\circ}$

ЂЕ乙ち $L L$

I6LF・28

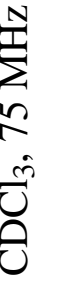

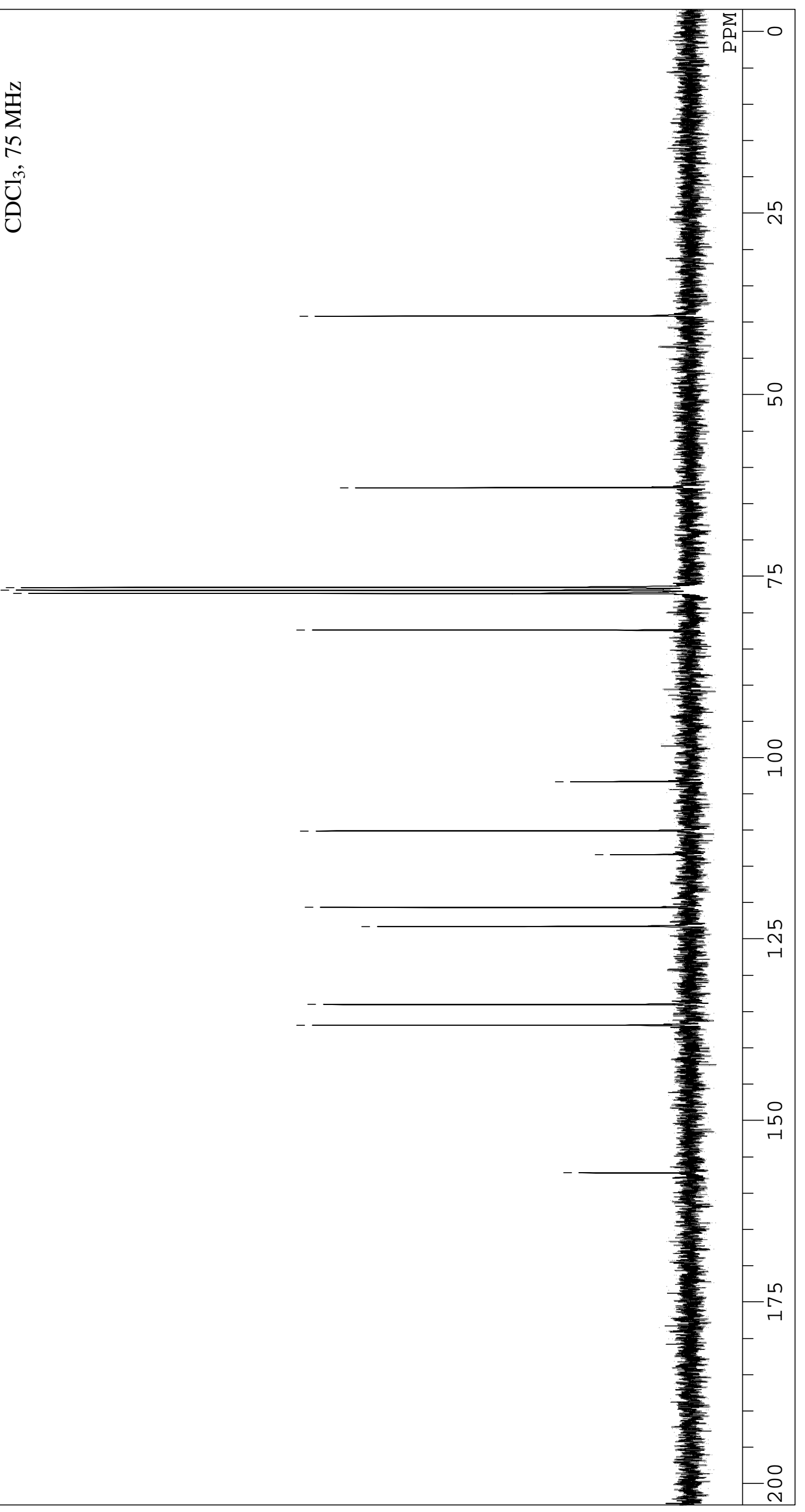

$\angle Z 9 Z \cdot \angle S I-$

бZL9・0ZI-
$686 Z \cdot \varepsilon Z \tau-$

ЂZL9 $0 Z I-$
$686 Z \cdot \varepsilon Z \tau-$

$9 \tau 90 * t \varepsilon \tau-$
9 $96^{\circ} \cdot 9 \varepsilon \tau-$

$9 \tau 90 * t \varepsilon \tau-$
9 $96^{\circ} \cdot 9 \varepsilon \tau-$

$\varepsilon \subseteq 乙 \tau \cdot 0 \tau]-$

$0 \varepsilon \sigma \varepsilon \cdot \varepsilon \tau T-$ 


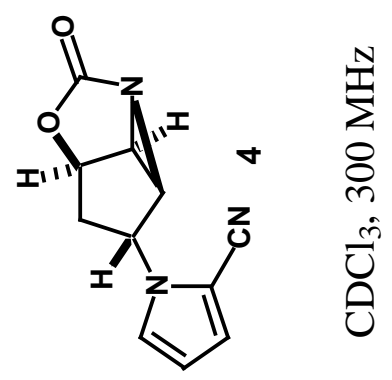

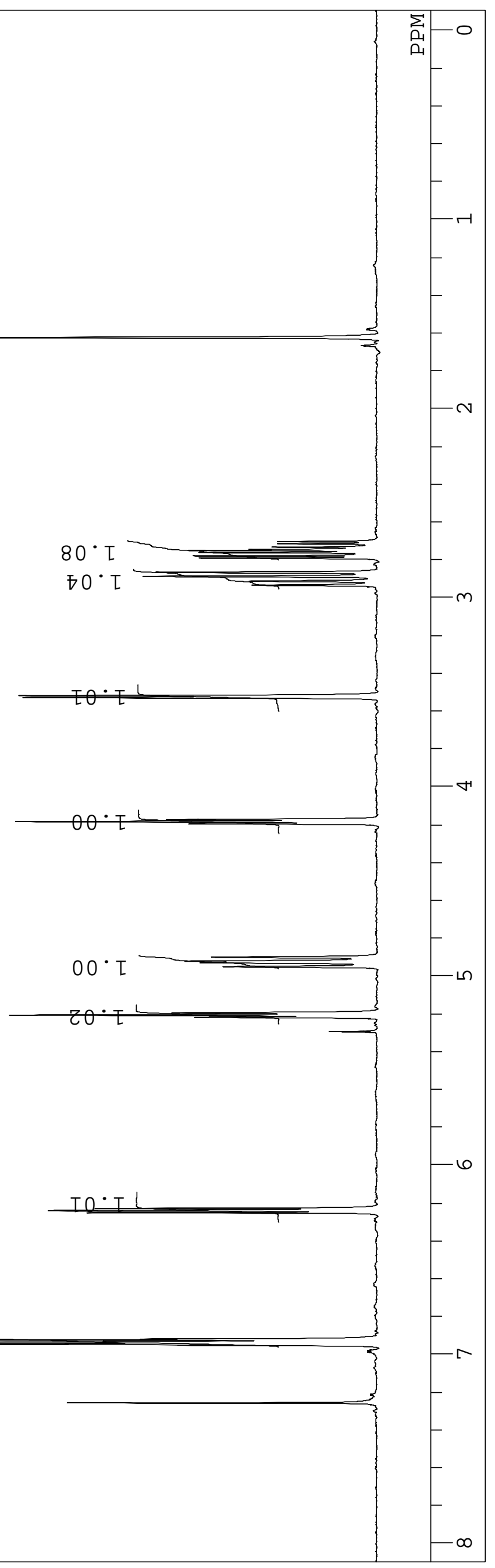


(1)

$\varepsilon 096^{\circ} 87 \longrightarrow$

โح9E $\varepsilon^{\circ} \varepsilon S \longrightarrow$

ง069.ง

$8898^{\circ} 99^{-}$

OIES $9 L$

$0000^{\circ}$

$069 \sigma^{\circ} L L$

$6 \angle 8 I^{\circ}$ โ 8

ந0ரL・IOL—

$\varepsilon \tau \varepsilon 乙 \cdot 0 \tau \tau-$

IฤIS ${ }^{\circ} \varepsilon I L$

$\varepsilon L \varepsilon L \cdot 乙 て T-$

乙8โ8.9Zโ—

$L \varepsilon 60^{\circ} \varepsilon 9 \mathrm{~L}$

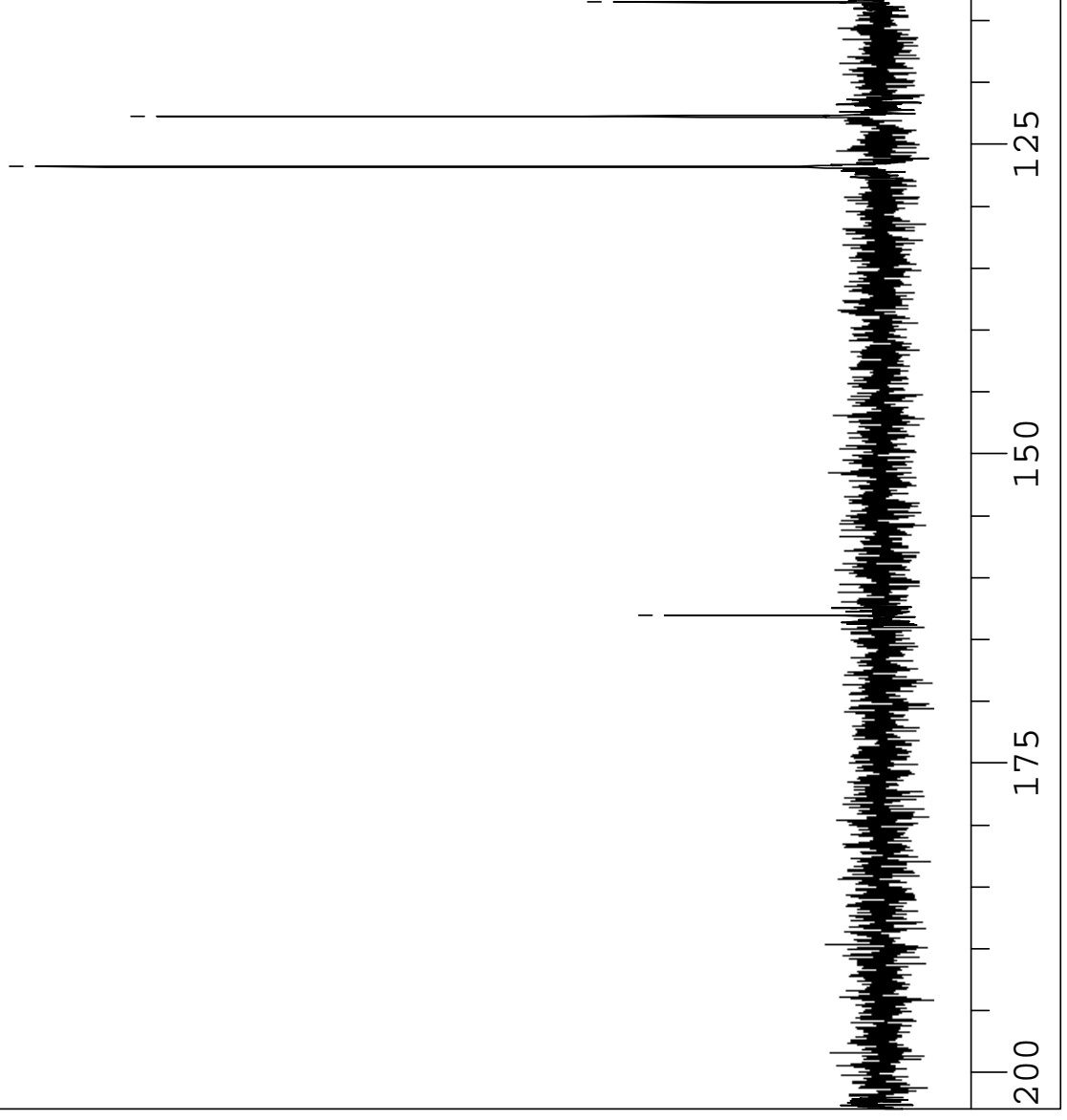


(1)

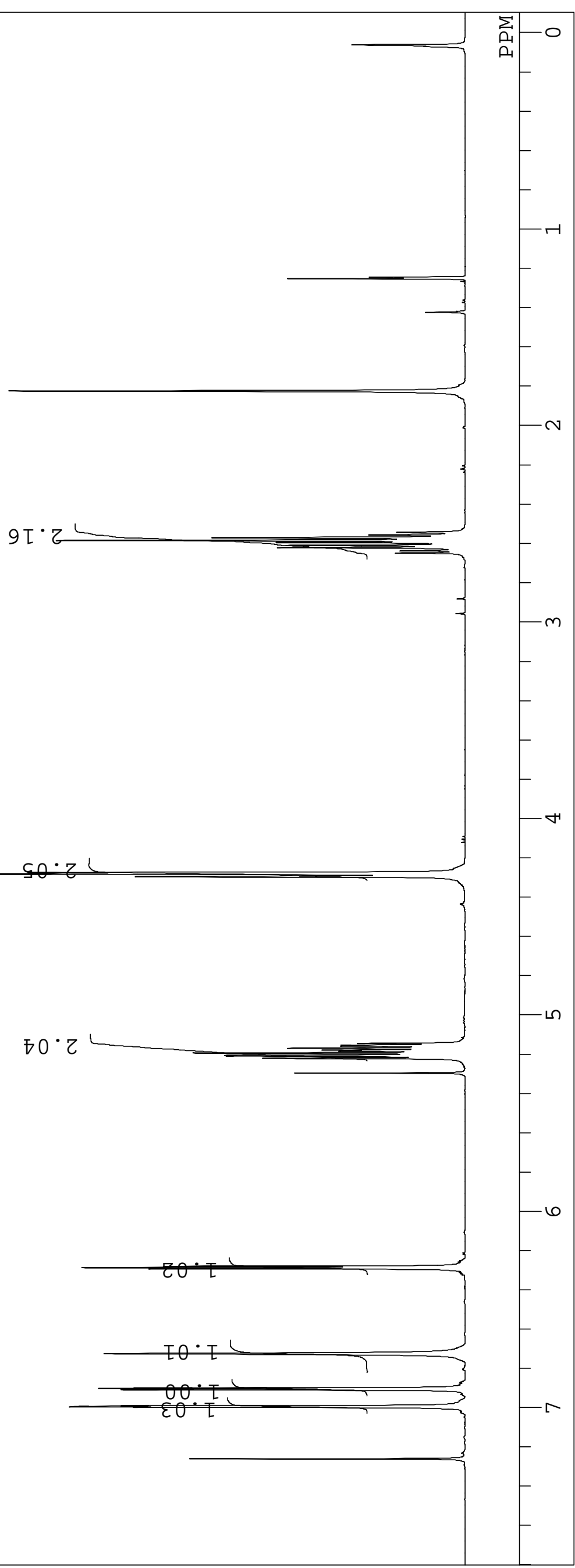




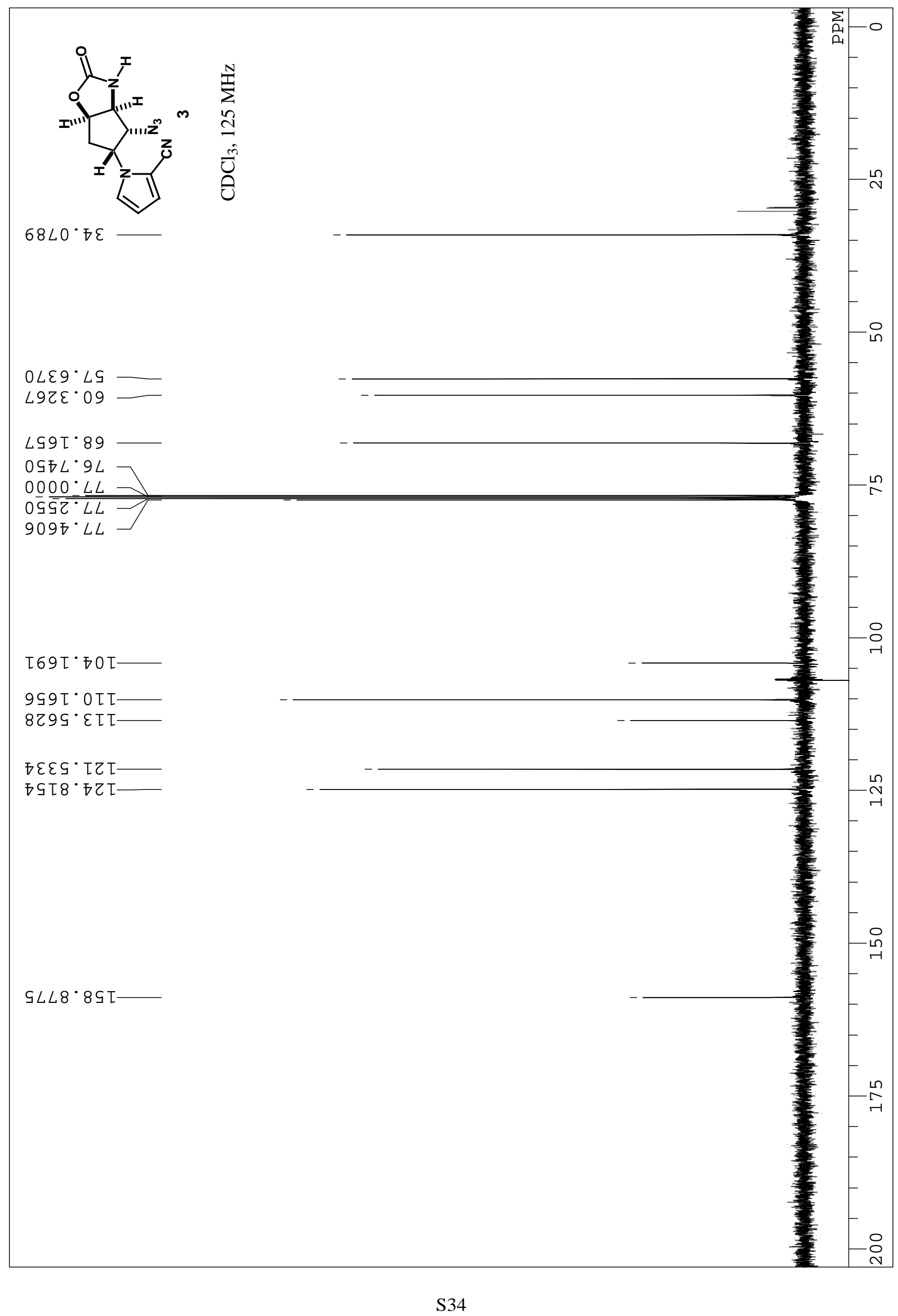



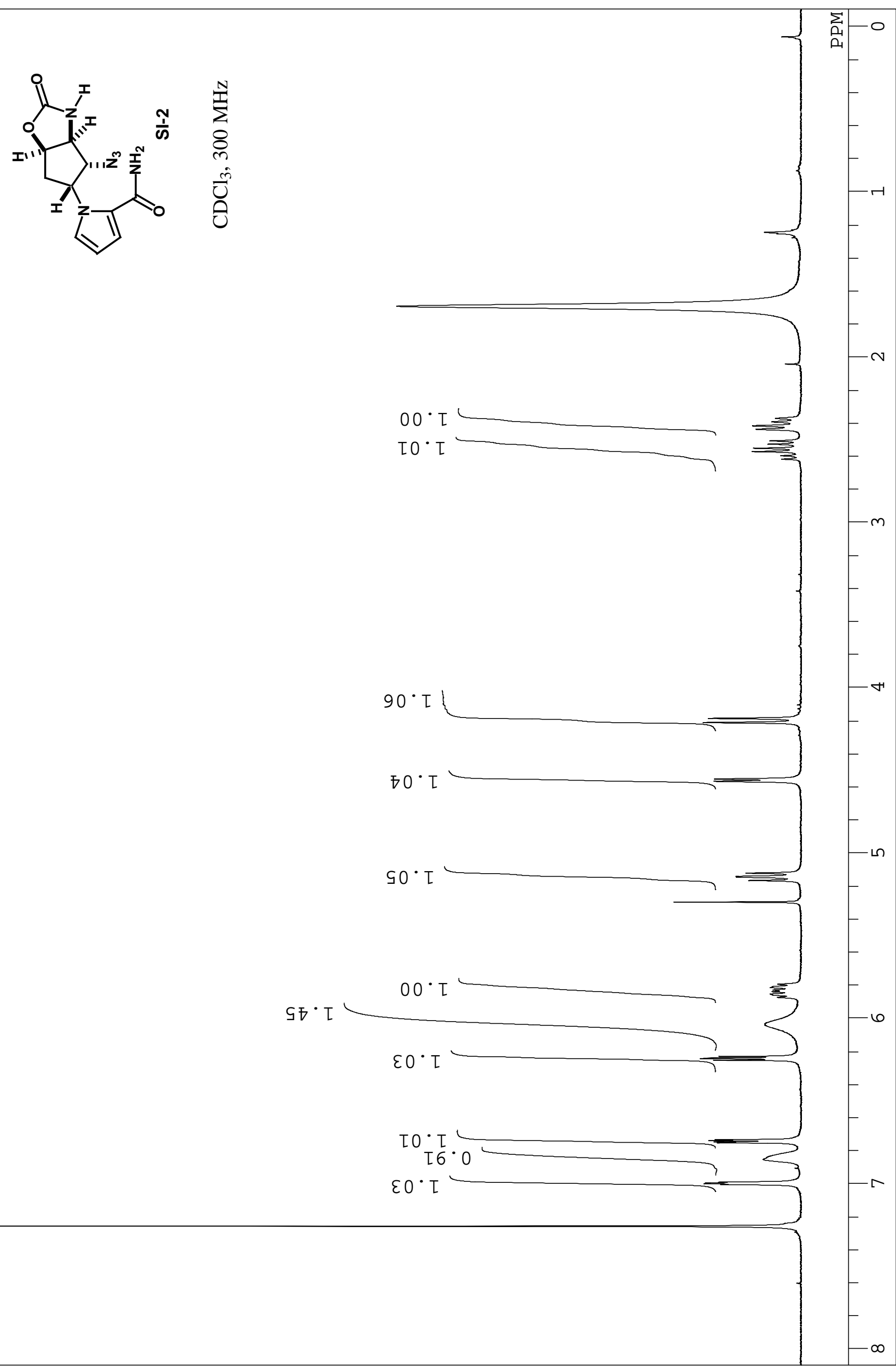


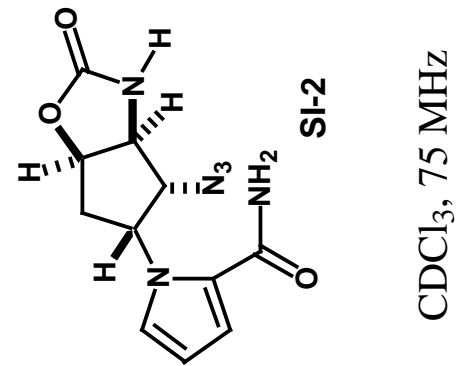

$9678 \cdot \varepsilon \varepsilon$

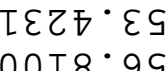

I9 I0*09

$9\left[67^{\cdot} 89\right.$

SZLG'9L

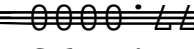

$\varepsilon 6\left[\nabla^{\bullet} L L\right.$

$\varepsilon 009^{\bullet} 80 \tau$

9てォて・GIL

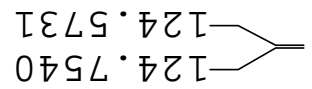

ஏてโォ・6S【—

$6 L \varepsilon 乙 \cdot \nabla 9 \tau$

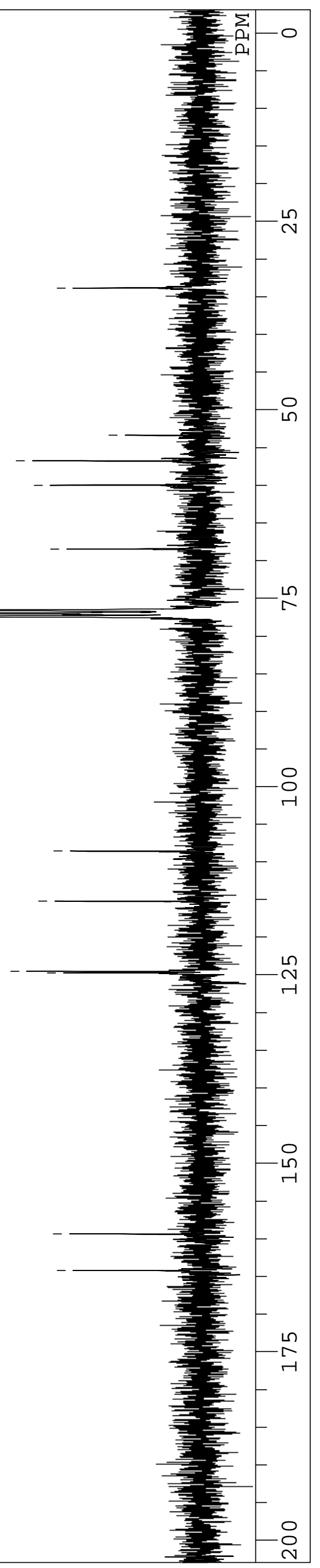


(1)

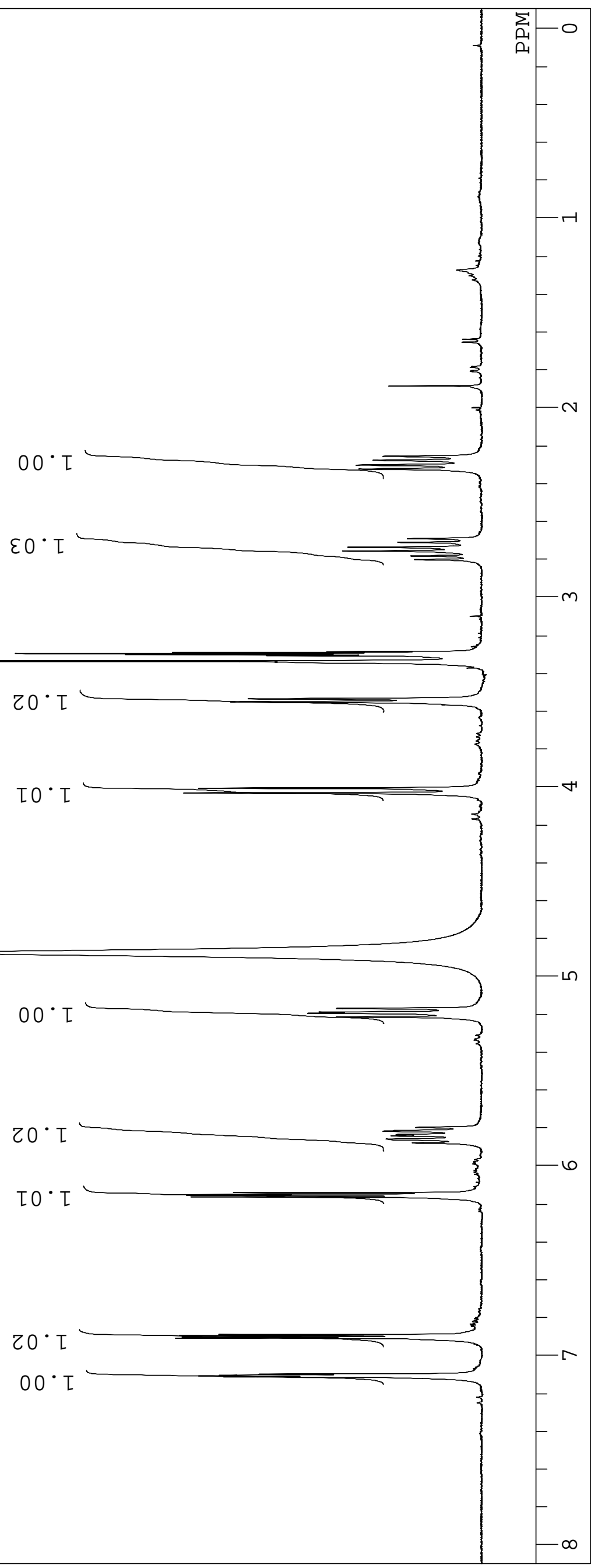




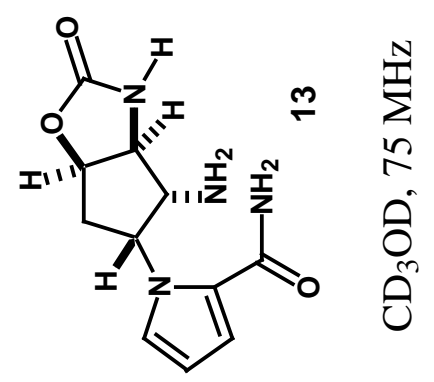

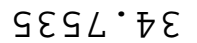

OS T $8 \mathrm{D}$

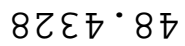

$\varepsilon Z \tau L \cdot 8 \hbar$

$0000 \cdot$

$96 L Z \cdot 67$

ZL9S.67

$\angle 978^{\circ} 67$

$88 \varepsilon 6^{\circ} 8 \mathrm{~s}$

8Sโ9.09

ऽऽE๐ $\varepsilon 9$

$2988^{\circ} 6 L$

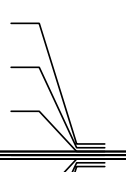

อิ

$-1$

$-\sqrt{ }$

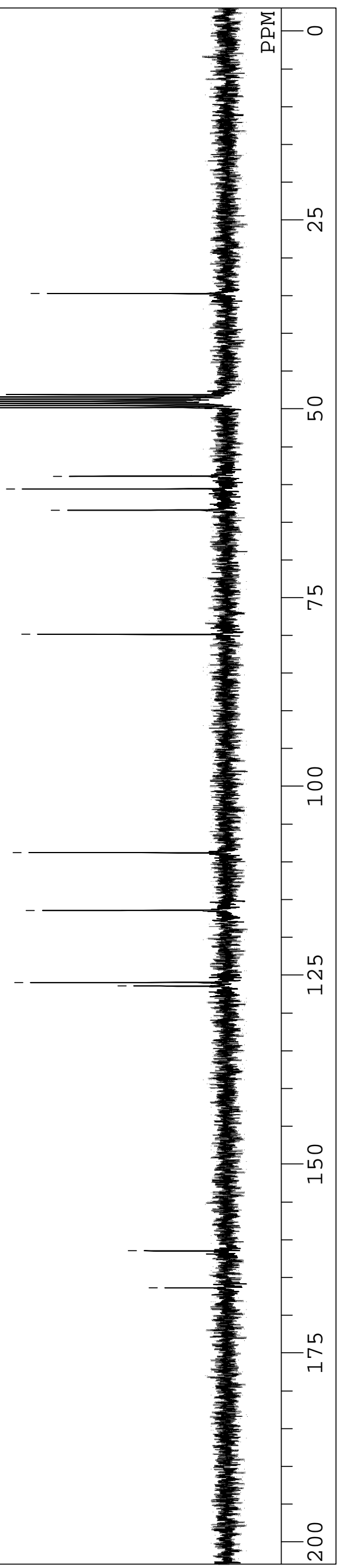

6ฤて与・โ9 I—

$0807 \cdot 99$ L

$98 \angle 6^{\circ} 92 I \longrightarrow$
ZLDV.9ZI $=$

$9908 \cdot 80 \tau$

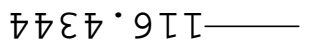




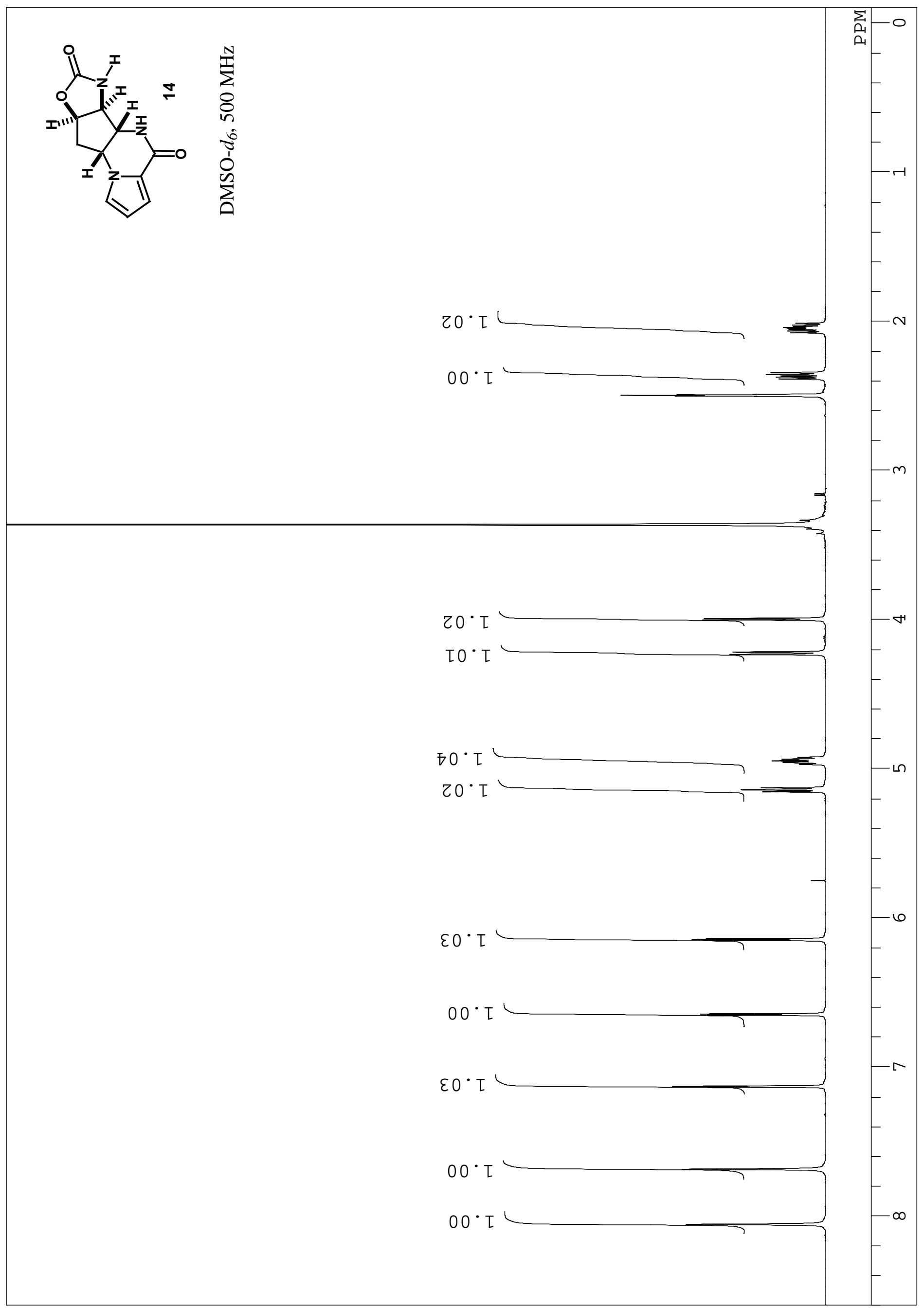




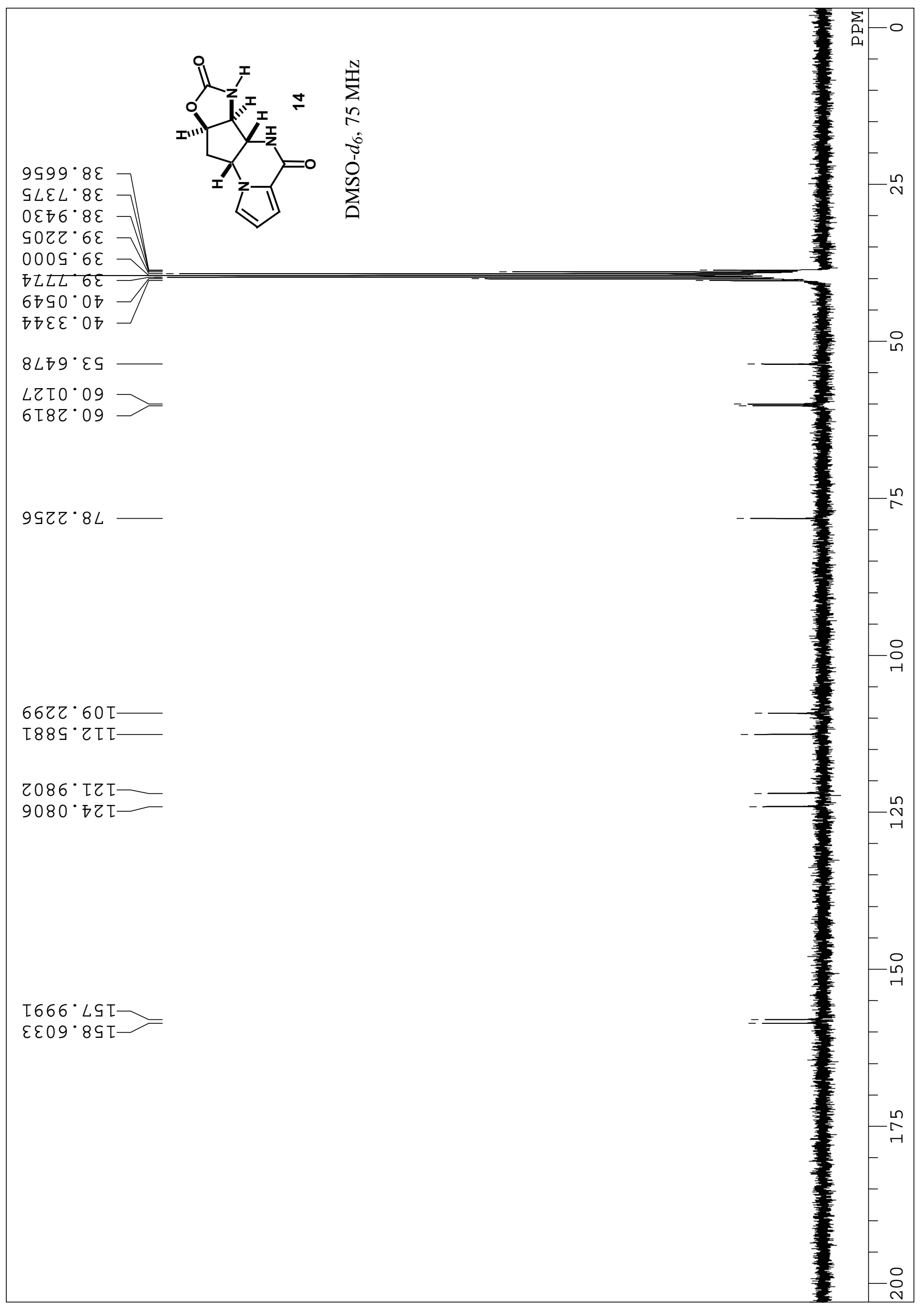


(1)
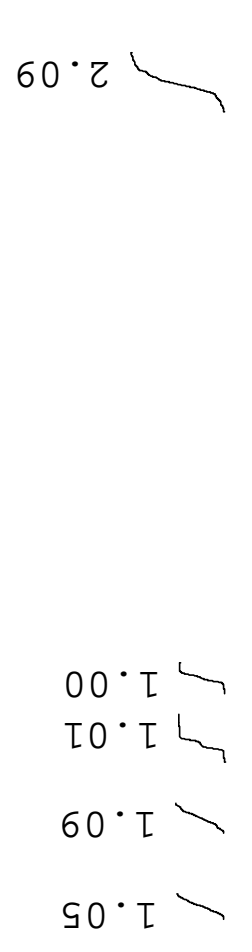


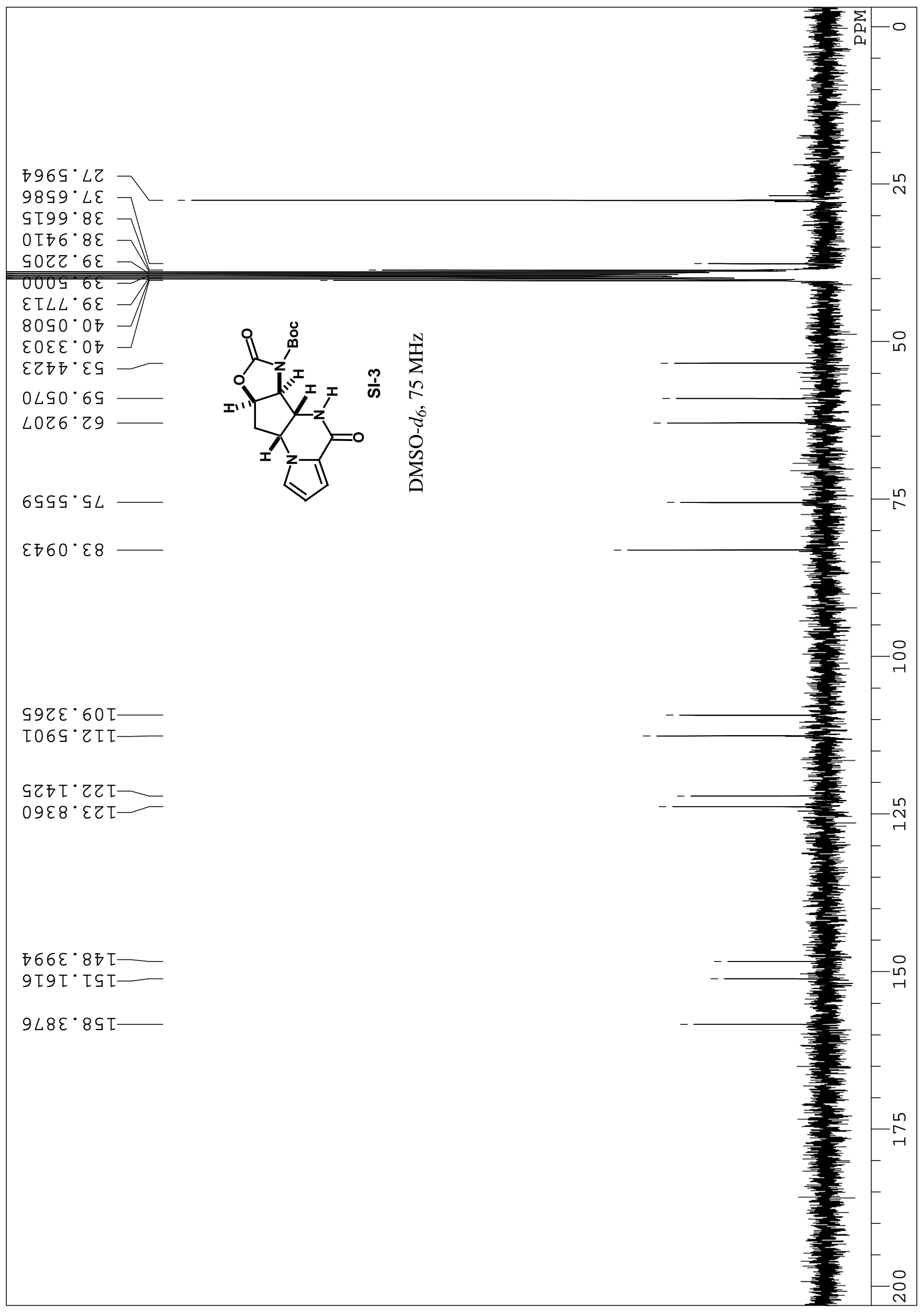



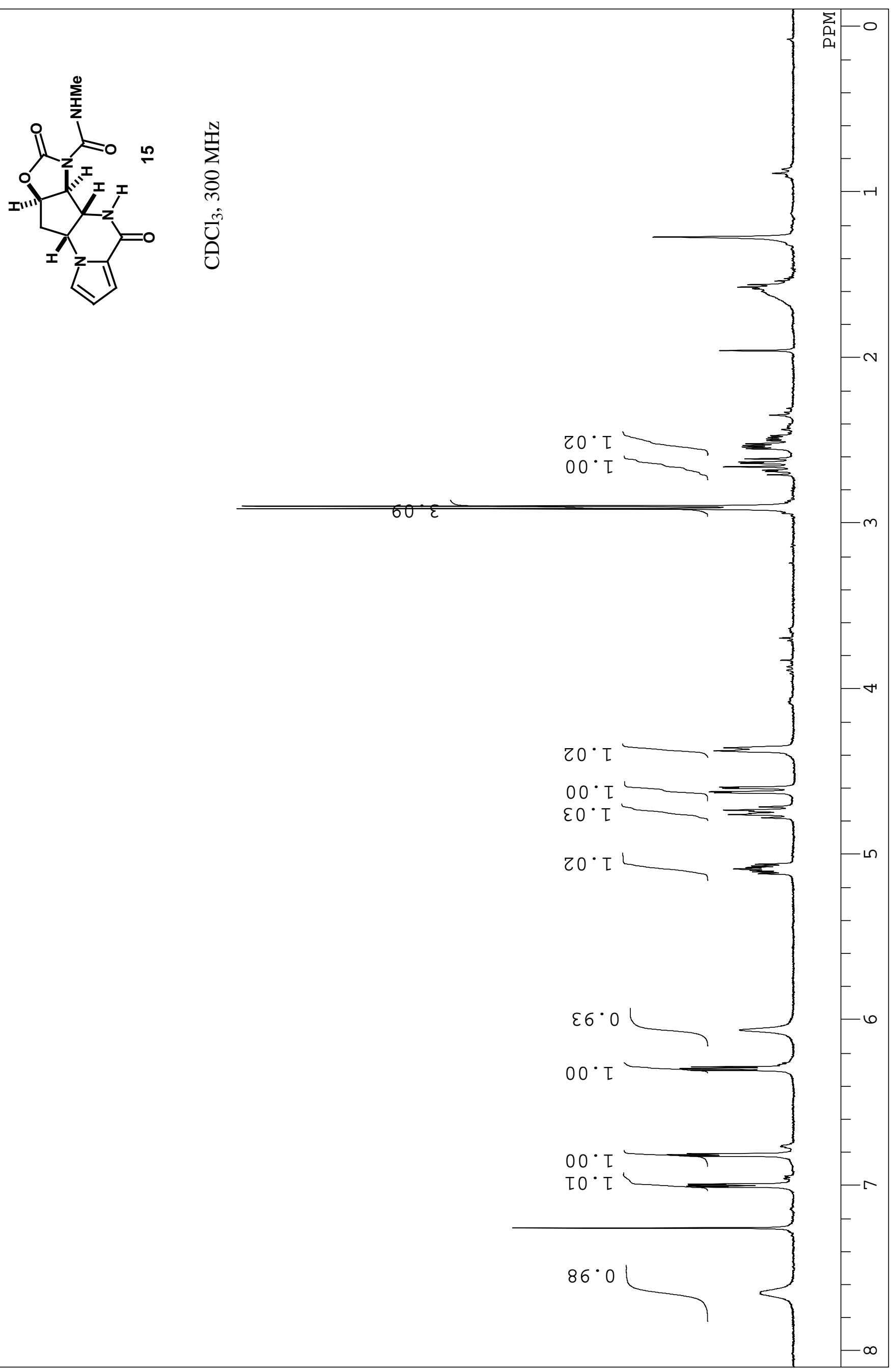


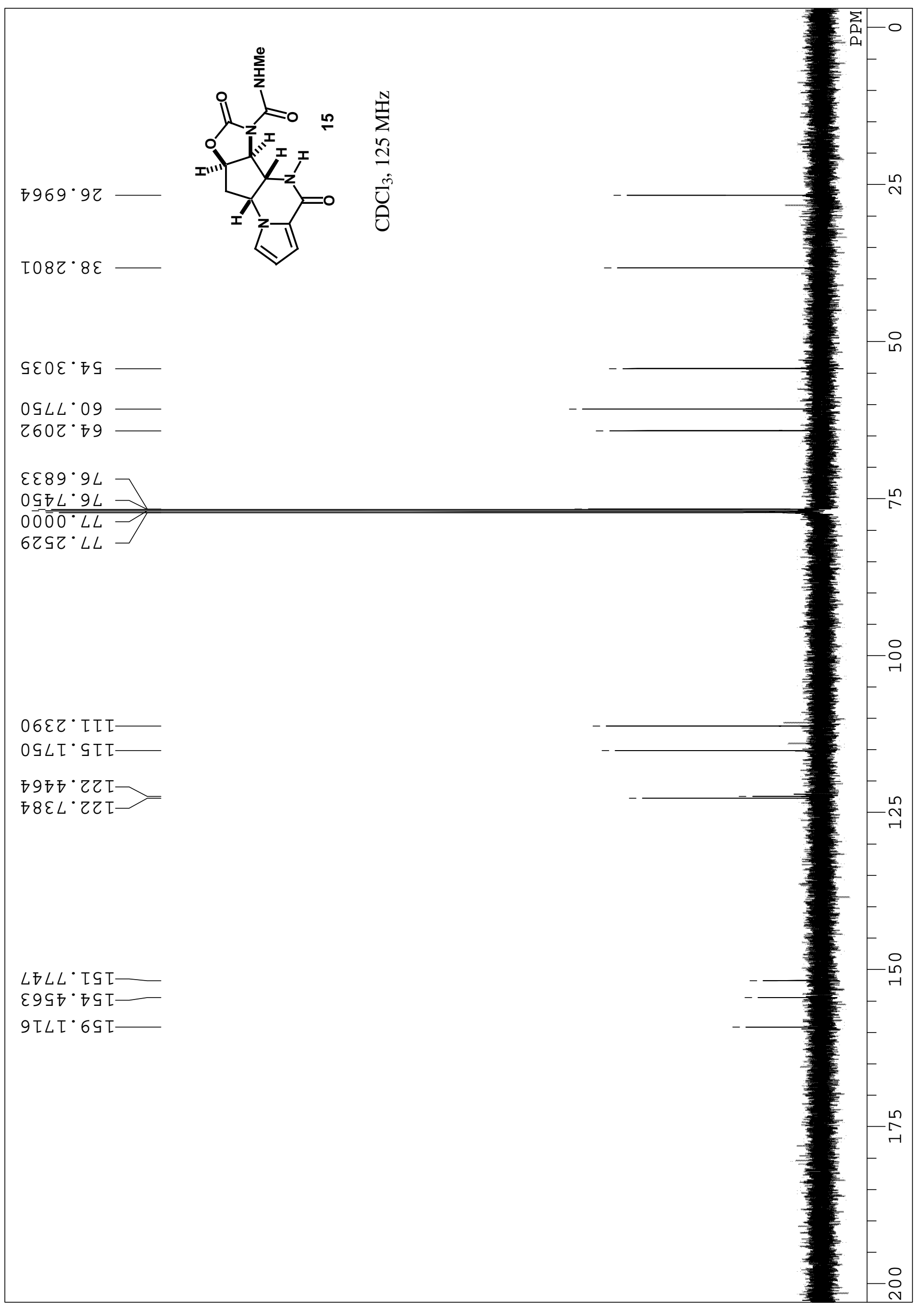




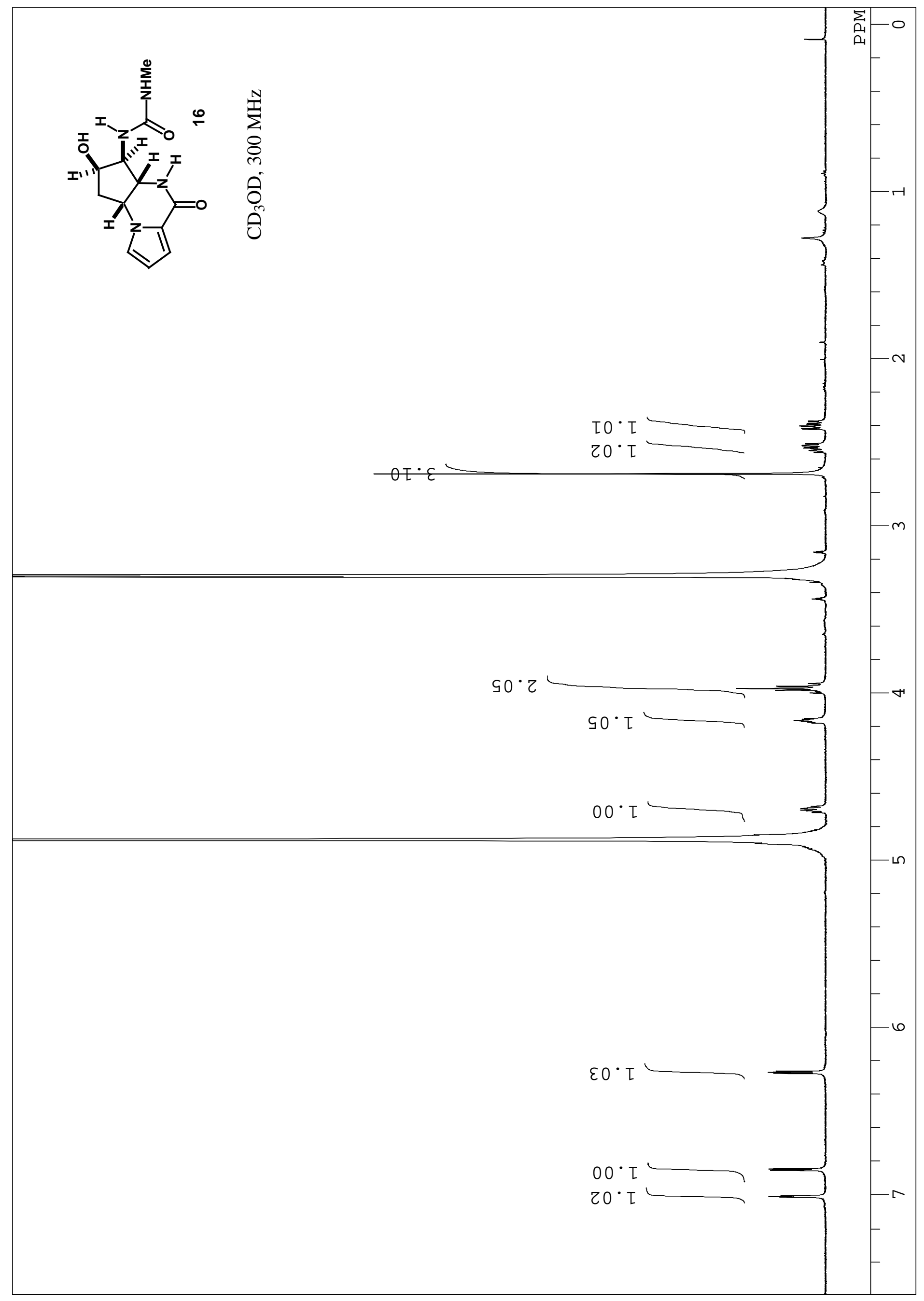




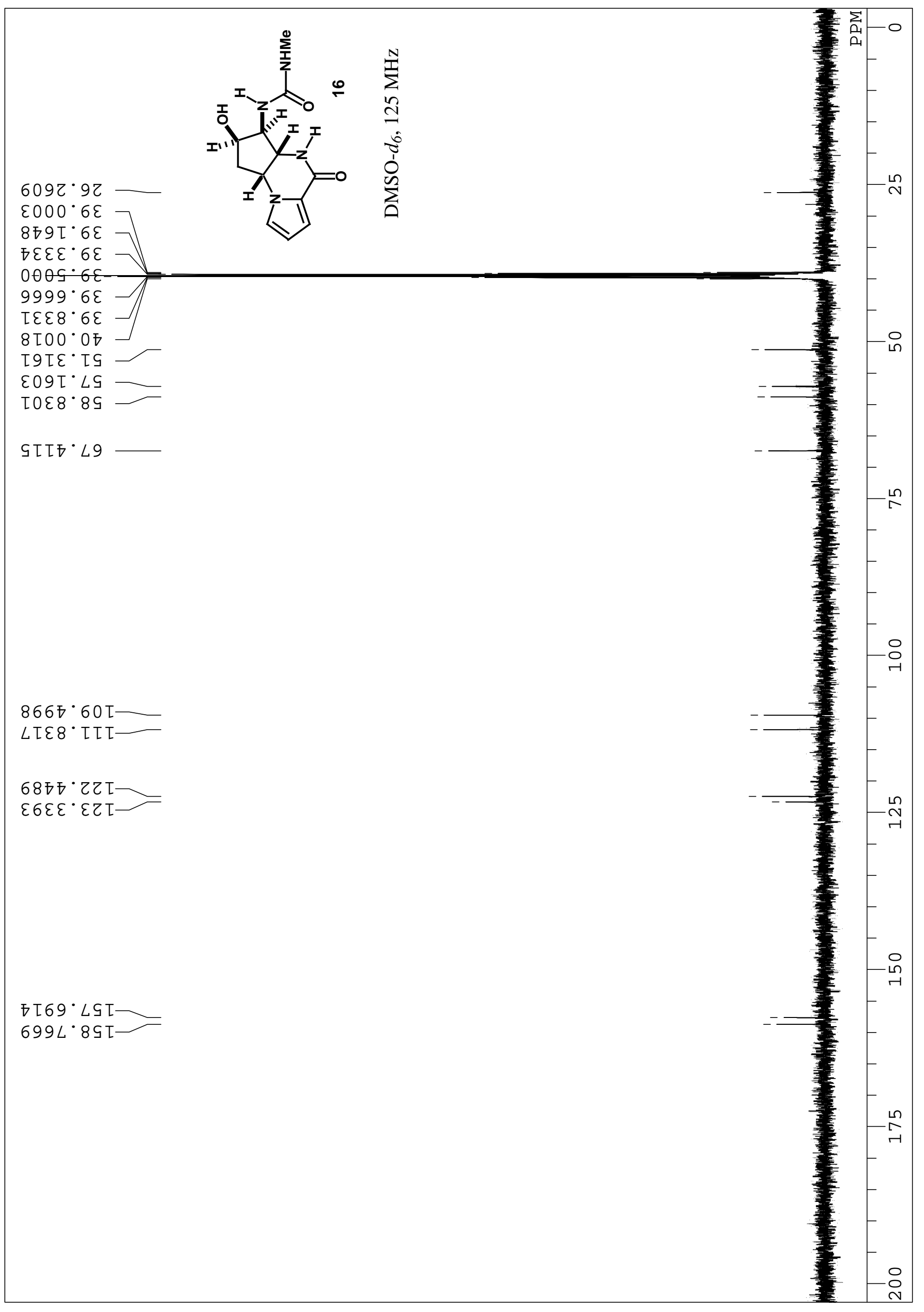




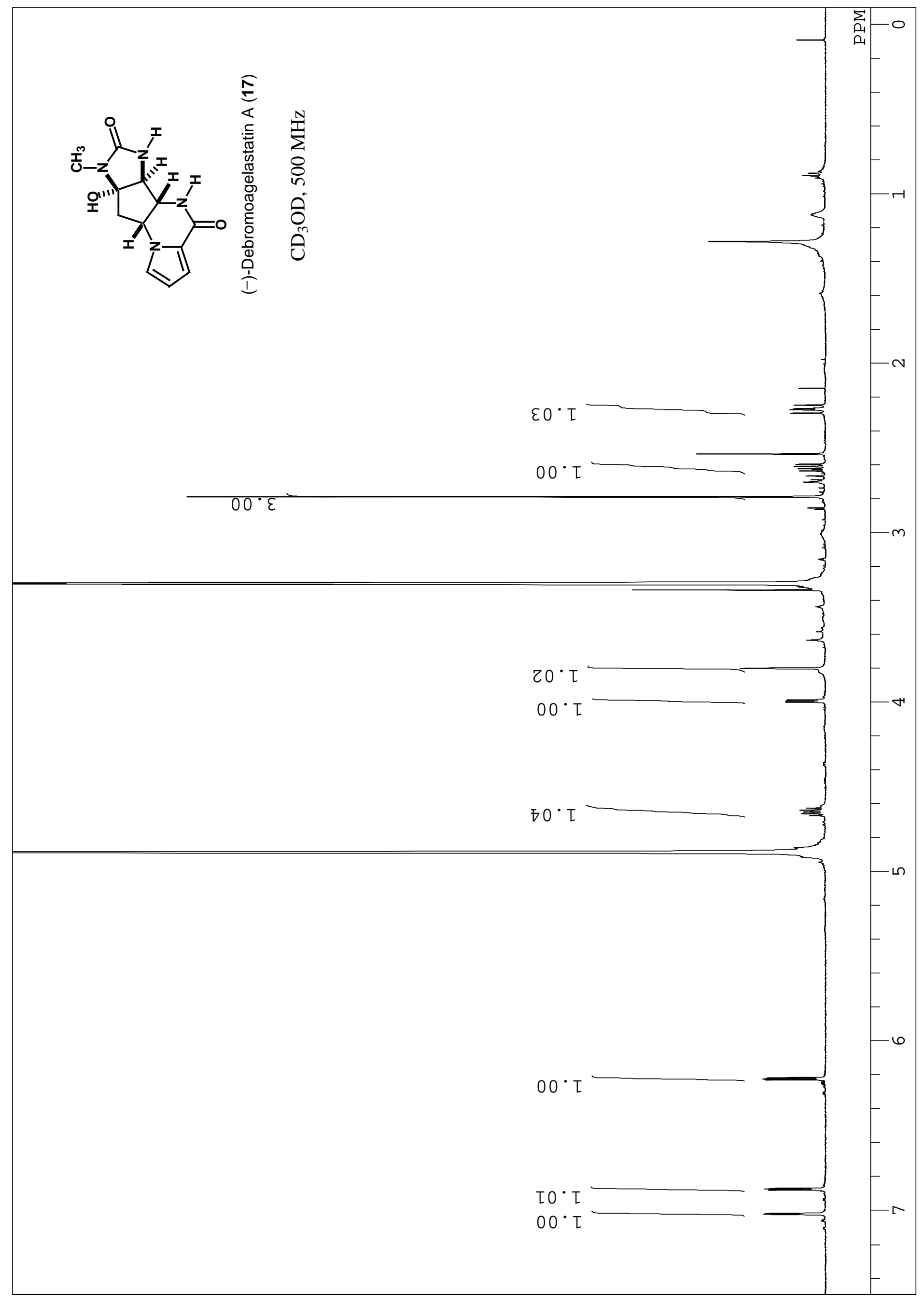


60

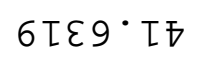

$006 \sigma^{\circ} 8 \mathrm{~T}$

$\angle 099 \cdot 8 \mathrm{~F}$

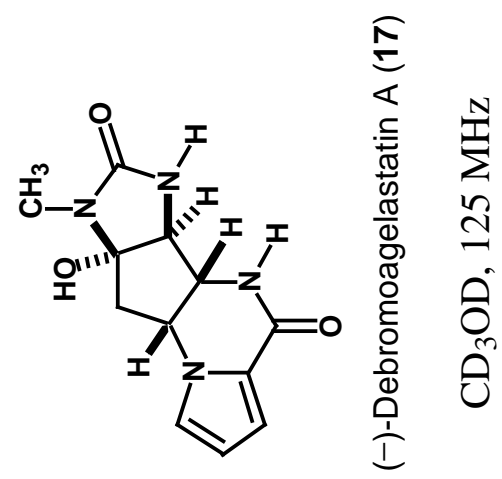

†โع8.87

$0000 \cdot 67$

$\angle O L I \cdot 67 \geqslant$

$8982 \cdot 6 \overline{1}$

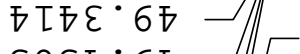

ง9S๐.67-

OZIS. 6 D

ZLZ9.6

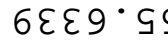

$\varepsilon 998^{\circ} \mathrm{Z9}$

$6266^{\circ} \angle 9$

โ8โ8・96
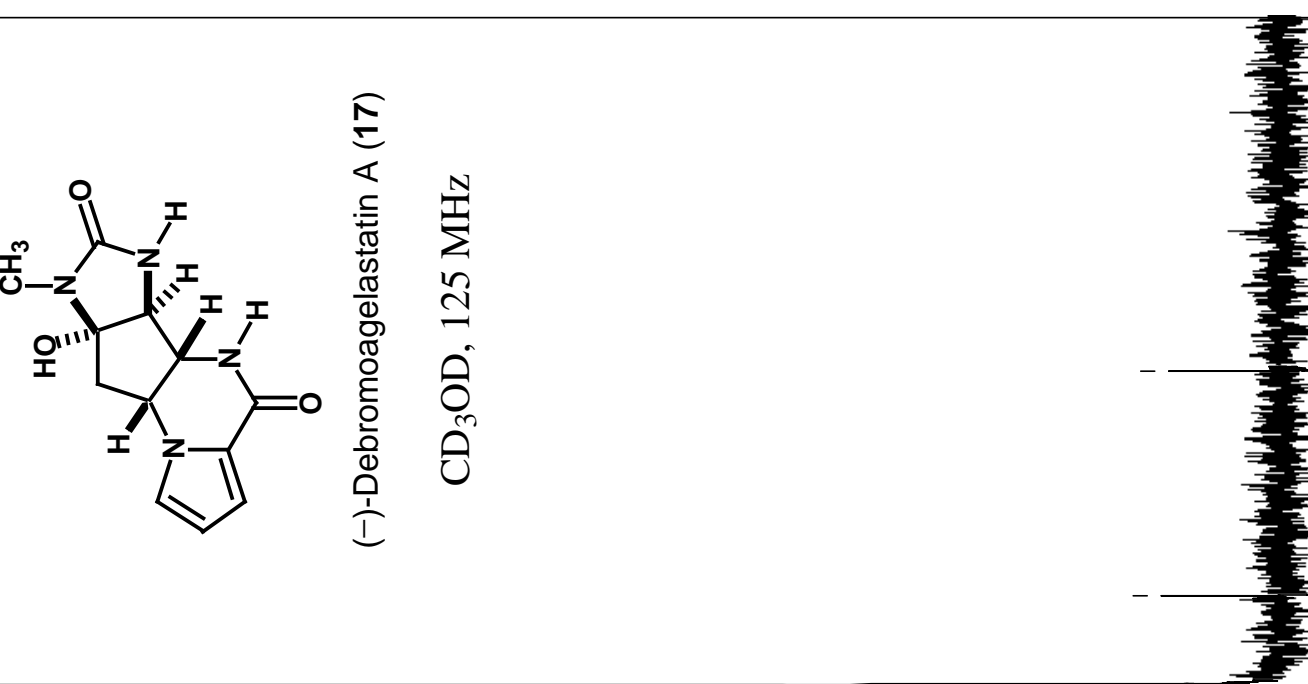

$\angle O L \cdot I \tau \tau$

โもEあ・SIL—

โもட8・ててโ—

โてゅ $9^{\circ}$ ऽて

โLย

oร90. 29 —

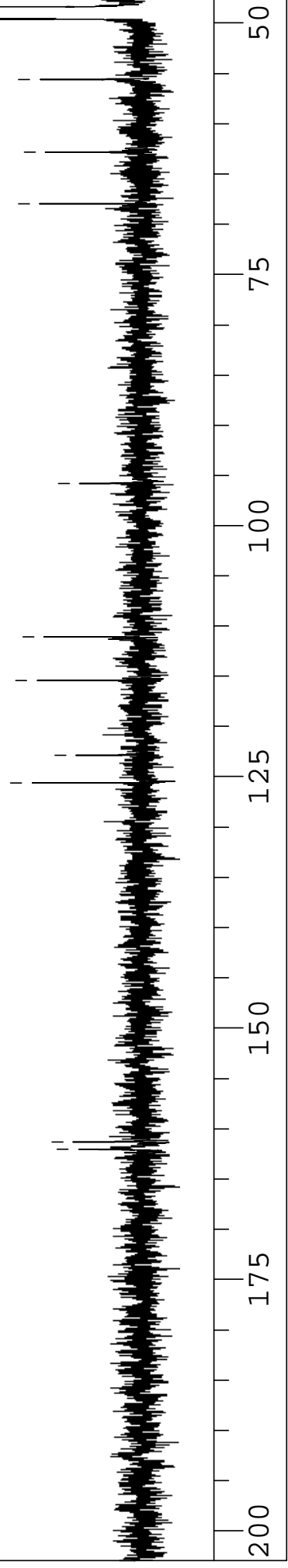




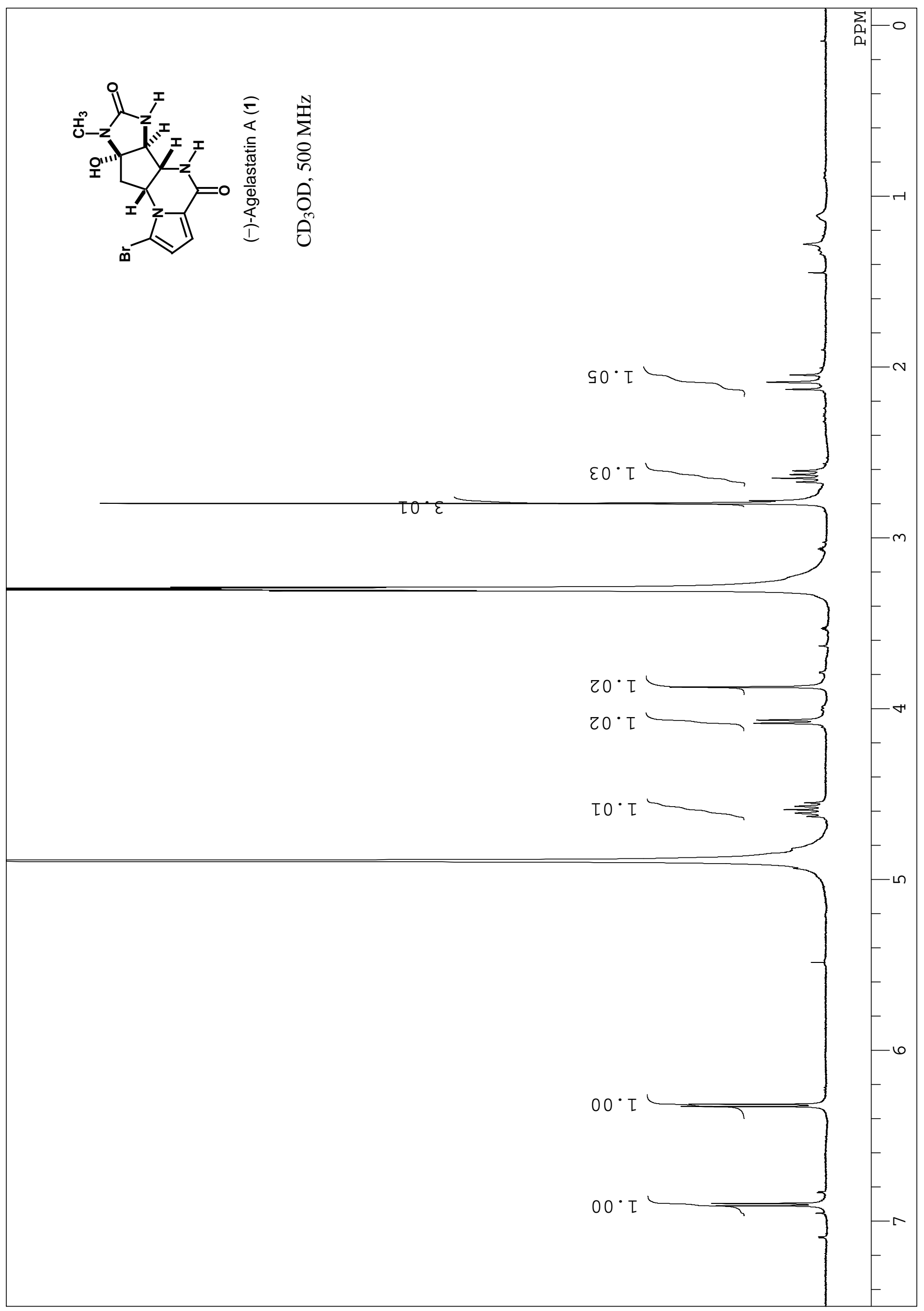




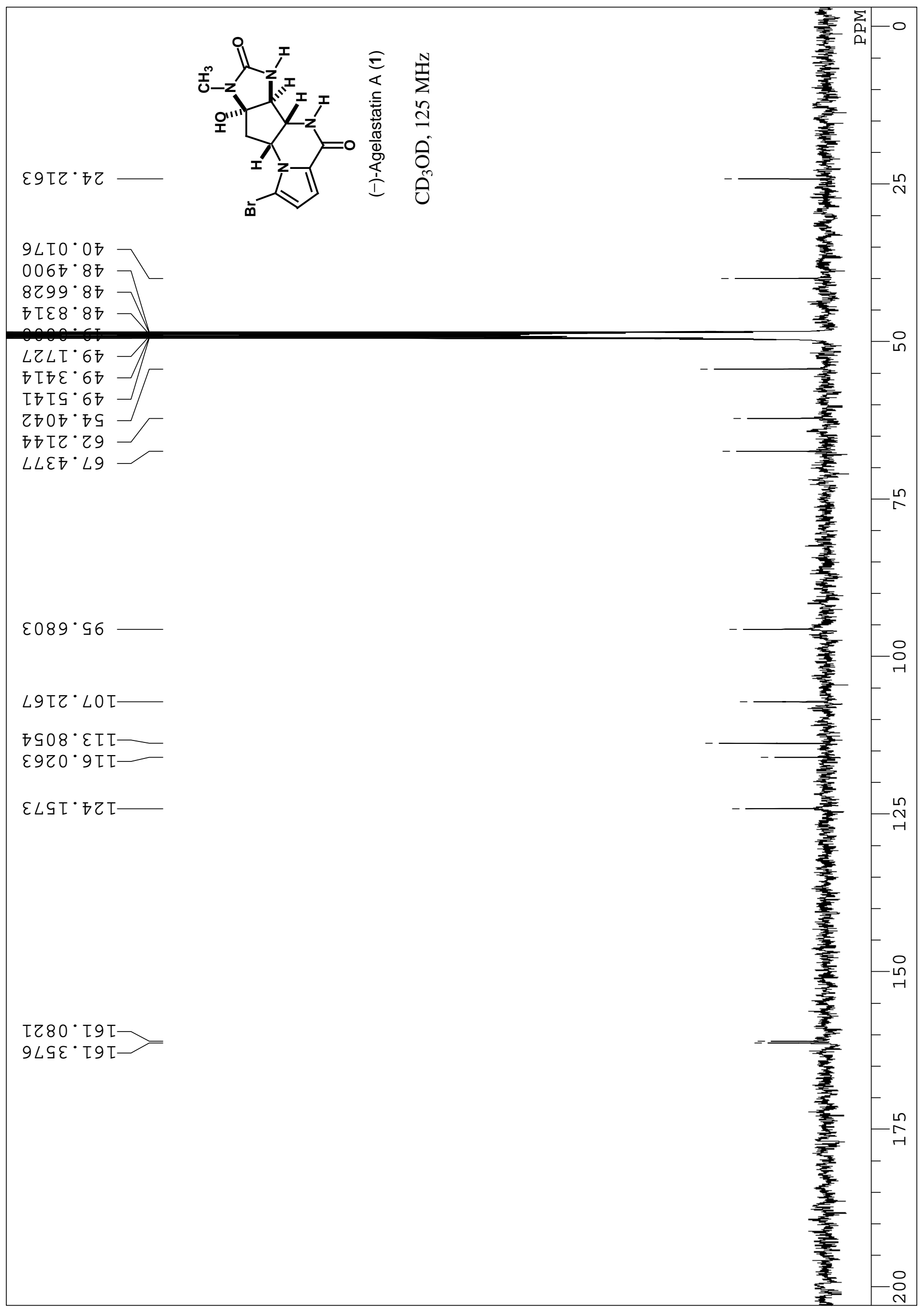

